\title{
Eigenvalue Asymptotics of Schrödinger Operators with Only Discrete Spectrum
}

\author{
By \\ Kazuya TaChizawa*
}

\section{$\S 1$. Introduction}

The present paper is devoted to the study of eigenvalue asymptotics of Schrödinger operators with only discrete spectrum. Let $V \in L_{\mathrm{loc}}^{\infty}\left(\boldsymbol{R}^{n}\right)$ and $V(x) \geq 1$. The Schrödinger operator $-\Delta+V$ admits the unique self-adjoint realization in $L^{2}\left(\boldsymbol{R}^{n}\right)$ where $\Delta$ denotes the Laplacian in $\boldsymbol{R}^{n}$. We denote it by $T$. If $V(x) \rightarrow \infty$ as $|x| \rightarrow \infty$, then $T$ has an infinite sequence of positive eigenvalues, $\left\{\lambda_{j}\right\}_{j=1}^{\infty}$, diverging to infinity. Let $N(\lambda), \lambda>0$, denote the number of eigenvalues less than $\lambda$ with repetition according to the multiplicities. Under suitable assumptions on $V$, we can prove the asymptotic formula

$$
N(\lambda) \sim(2 \pi)^{-n} \omega_{n} \int_{R^{n}}(\lambda-V(x))_{+}^{n / 2} d x \quad \text { as } \quad \lambda \rightarrow \infty
$$

where $\omega_{n}$ is the volume of the unit ball in $\boldsymbol{R}^{n}$ and

$$
f(x)_{+}=\max \{f(x), 0\} .
$$

For the results of the form of (1.1) we refer to Edmunds and Evans [2], Feigin [4], Fleckinger [5], Fleckinger and Lapidus [6, Section 5], Levendorskii [9], Reed and Simon [10, Theorem XIII. 81], Rozenbljum [12], Tamura [16], Titchmarsh [17, Chapter XVII] and de Wet and Mandl [18].

Communicated by S. Matsuura, March 25, 1991. Revised May 1, 1992.

1991 Mathematics Subject Classification: 35P20

* Mathematical Institute, Tôhoku University, Sendai 980, Japan. 
In the present paper we will give a new criteria on the potential $V$ for the formula (1.1). Our main results are Theorems 2.1, 2.2 and 2.3 and those are proved by Dirichlet-Neumann bracketing method and a modification of results in Fleckinger and Lapidus [7]. By virtue of these main results we can establish the formula (1.1) for several potentials.

First we will consider radial potentials, that is, $V(x)=f(|x|), f \in C^{1}([0$, $\infty)), f$ is strictly increasing, $f \geq 1$, and $f(t) \rightarrow \infty$ as $t \rightarrow \infty$. If $f$ satisfies the following condition

$$
\lambda^{n / 2} f^{-1}(\lambda)^{n-v_{n}}=o\left(\int_{0}^{f^{-1}(\lambda)}(\lambda-f(t))^{n / 2} t^{n-1} d t\right) \text { as } \lambda \rightarrow \infty
$$

where $v_{n}=1 /(2 n)(n \geq 3), v_{2}=v_{1}=1 / 8$, then we have the formula (1.1).

The condition (1.2) is satisfied, for example, if $f \in C^{2}([0, \infty))$ and $f^{\prime \prime} \geq 0$. Also we can prove (1.2) for very slowly growing function such as $f(t)=\log \log \cdots \log t$ for large $t$. Previously, the eigenvalue asymptotics for these very slowly growing potentials on $\mathbb{R}^{n}$ are studied only by Levendorskii [9, p177, Theorem 5] for $C^{\infty}$ potentials and our theorem gives the formula (1.1) for $C^{1}$ potentials.

Next we will consider potentials which are not necessarily radial. Our main theorem gives the following new result. If $V \in C^{1}\left(\mathbb{R}^{n}\right)(n \geq 3), V \geq 1$, $V(x) \rightarrow \infty$,

$$
|\nabla V(x)| \leq c V(x)^{1+a}(0 \leq a<1 / 2)
$$

for large $|x|$ and $\sigma(2 \lambda) \leq c \sigma(\lambda)$ for large $\lambda$ where $\sigma(\lambda)=\left|\left\{x \in \mathbb{R}^{n}: V(x)<\lambda\right\}\right|$, then we have the formula (1.1). The condition (1.3) is studied by Feigin [4] (cf. [9, p178]) for $C^{\infty}$ potentials and our requirement on the regularity of potentials is $C^{1}$-regularity.

Also we will give an extention of the result by Titchmarsh [17, p176] and alternative proofs of results by Rozenbljum [12, Theorem 2] and Fleckinger [5].

Furthermore we will give asymptotic formulas for some nonclassical potentials. A nonclassical potential is the potential whose zero set is an unbounded subset, for example, $V(x, y)=|x y|^{\alpha}$ on $\mathbb{R}^{2}$. The nonclassical potentials are studied by Gurarie [8], Levendorskii [9], Robert [11], Simon [13], Solomyak [14], and Tachizawa [15], and they only consider potentials whose zero sets are cones. Our results contain new potentials whose zero sets are not cones such as $V(x, y)=\Pi_{i=1}^{p}\left|x-a_{i}\right|^{\alpha_{i}} \Pi_{j=1}^{q}\left|y-b_{j}\right|^{\beta_{j}}$ on $\mathbb{R}^{2}$. The 
results on these nonclassical potentials will be proved by means of simple modifications of the main results.

We will outline the content of this paper. We will state our main results in Section 2 and its proofs will be given in Section 3. In Section 4 there are some applications of the main results. In Section 5 we will apply our method to some examples of Schrödinger operators with nonclassical potentials.

\section{Acknowledgement}

The author would like to thank Professor Satoru Igari for many useful comments.

\section{§2. Main Results}

Let $\Omega$ be a measurable subset in $R^{n}$. We denote the boundary, the closure and the $n$-dimensional Lebesgue measure of $\Omega$ by $\partial \Omega, \bar{\Omega}$ and $|\Omega|$, respectively. For $p(1 \leq p \leq \infty) L^{p}(\Omega)$ denotes the usual Lebesgue space with the norm \|\|$_{p, \Omega}$. When $\Omega$ is an open subset in $\mathbb{R}^{n}, L_{\text {loc }}^{1}(\Omega)\left(L_{\text {loc }}^{1}(\bar{\Omega})\right)$ denotes the space of all measurable functions which are Lebesgue integrable on every compact (bounded) subset of $\Omega$. The definitions of $L_{\mathrm{loc}}^{\infty}(\Omega)$ and $L_{\mathrm{loc}}^{\infty}(\bar{\Omega})$ are similar.

Let $V(x) \in L_{\mathrm{loc}}^{\infty}\left(\mathbb{R}^{n}\right), \quad V \geq 1$ and $V(x) \rightarrow \infty$ as $|x| \rightarrow \infty$. Consider the sesquilinear form

$$
t(u, v)=\int_{\boldsymbol{R}^{n}} \nabla u \overline{\nabla v} d x+\int_{\boldsymbol{R}^{n}} V u \bar{v} d x
$$

for $u, v \in C_{0}^{\infty}\left(\boldsymbol{R}^{n}\right)$ where $\nabla u$ denotes the gradient of $u$. Then $t$ is a sesquilinear form in $L^{2}\left(\boldsymbol{R}^{n}\right)$ with the domain $C_{0}^{\infty}\left(\mathbb{R}^{n}\right)$.

Let $H(V)$ be the closure of $C_{0}^{\infty}\left(\boldsymbol{R}^{n}\right)$ by the norm $t(u, u)^{1 / 2}$. Then $H(V)$ is a Hilbert space with the inner product $t(\cdot, \cdot)$. We can easily show that $t$ is closable in $L^{2}\left(\boldsymbol{R}^{n}\right)$ and let $\tilde{t}$ be the closure of $t$ to $H(V)$. Then $\tilde{t}$ is a closed, non negative, and symmetric sesquilinear form in $L^{2}\left(\boldsymbol{R}^{n}\right)$ with the domain $H(V)$. By the representation theorem (cf. [3, Chapter IV, Theorem 2.4]), there exists a self-adjoint operator $T$ in $L^{2}\left(\mathbb{R}^{n}\right)$ with the domain $D(T)$ which 
is a dense subset of $H(V)$ such that

$$
\tilde{t}(u, v)=(T u, v)
$$

for all $v \in H(V)$ and all $u \in D(T)$ where

$$
\begin{aligned}
D(T)=\{u \in H(V): \tilde{t}(u, v)=(f, v) & \text { for some } f \in L^{2}\left(\mathbb{R}^{n}\right) \\
& \text { and for all } v \in H(V)\}
\end{aligned}
$$

and

$$
(u, v)=\int_{\boldsymbol{R}^{n}} u \bar{v} d x
$$

By the assumption on $V, T$ has only discrete spectrum (cf. [3, Chapter VIII, Section 4]) and we define $N(\lambda)$ as the number of eigenvalues of $T$ less than $\lambda>0$.

Here we introduce some notations and conditions.

Let $\lambda_{1}$ be a positive constant and $l$ be a function from $\left(\lambda_{1}, \infty\right)$ to $(0$, $\infty)$. For $\lambda>\lambda_{1}$, we consider a tessellation of $\mathbb{R}^{n}$ by the family of congruent cubes $\left\{\bar{Q}_{\zeta}\right\}_{\zeta \in Z_{\lambda}}$ where $Z_{\lambda}$ denotes an index set and $\left\{Q_{\zeta}\right\}_{\zeta \in Z_{\lambda}}$ are disjoint open cubes with side length $l(\lambda)$ whose sides are parallel to the coordinate axes.

We set

$$
I_{\lambda}=\left\{\zeta \in Z_{\lambda}: \text { ess } \sup _{x \in Q_{\zeta}} V(x)<\lambda\right\}
$$

and

$$
J_{\lambda}=\left\{\zeta \in Z_{\lambda}: \text { ess } \inf _{x \in Q_{\zeta}} V(x)<\lambda\right\}
$$

We consider the following conditions.

(H1) There exist a positive constant $\lambda_{2} \geq \lambda_{1}$ and a function $a_{1}:\left(\lambda_{2}\right.$, $\infty) \rightarrow(0,1)$ such that

$$
I_{\lambda} \neq \varnothing
$$


and

$$
\frac{\left(\#\left(J_{\lambda} \backslash I_{\lambda}\right)\right)}{\left(\# I_{\lambda}\right)} \leq a_{1}(\lambda)
$$

for all $\lambda>\lambda_{2}$ where $\# I_{\lambda}$ and $\#\left(J_{\lambda} \backslash I_{\lambda}\right)$ denote the number of elements in $I_{\lambda}$ and $J_{\lambda} \backslash I_{\lambda}$, respectively.

(H2) Let $p_{n}=n / 2(n \geq 3), p_{2}=2$, and $p_{1}=1$. Let

$$
g(x)=1-\frac{V(x)}{\lambda} \text { and } g_{\zeta}=\left(\frac{1}{\left|Q_{\zeta}\right|} \int_{Q_{\zeta}} g(x)^{n / 2} d x\right)^{2 / n}
$$

for $\zeta \in I_{\lambda}$. There exist positive constants $\gamma$ and $\lambda_{3} \geq \lambda_{1}$, and a function $a_{2}$ : $\left(\lambda_{3}, \infty\right) \rightarrow(0,1 / 2)$ such that

$$
G_{n}(V, \lambda) \leq \gamma \sigma(\lambda) a_{2}(\lambda)
$$

for all $\lambda>\lambda_{3}$ where

$$
G_{n}(V, \lambda)=l(\lambda)^{n\left(1-n /\left(2 p_{n}\right)\right)} \sum_{\zeta \in I_{\lambda}}\left(\int_{Q_{\zeta}}\left|g(x)-g_{\zeta}\right|^{p_{n}} d x\right)^{n /\left(2 p_{n}\right)}
$$

and

$$
\sigma(\lambda)=\left|\left\{x \in \mathbb{R}^{n}: V(x)<\lambda\right\}\right|
$$

The following theorem holds.

Theorem 2.1. Let $V \in L_{\mathrm{loc}}^{\infty}\left(\mathbb{R}^{n}\right), V \geq 1$ and $V(x) \rightarrow \infty$ as $|x| \rightarrow \infty$. Let $\lambda_{1}$ be a positive constant and $l$ be a function from $\left(\lambda_{1}, \infty\right)$ to $(0, \infty)$. Suppose that the conditions (H1) and (H2) hold.

Then there exist positive constants $\lambda_{0}$ and $c$ such that

$$
\begin{aligned}
& \left|N(\lambda)-(2 \pi)^{-n} \omega_{n} \int_{R^{n}}(\lambda-V)_{+}^{n / 2} d x\right| \\
& \leq c \sigma(\lambda)\left\{\lambda^{n / 2} a_{1}(\lambda)+\lambda^{n / 2} a_{2}(\lambda)^{\theta_{n}}+l(\lambda)^{-n}+\lambda^{(n-1) / 2} l(\lambda)^{-1}\right\}
\end{aligned}
$$


for all $\lambda>\lambda_{0}$ where $\theta_{n}=\min \{1 / 2,1 / n\}$ and $c$ depends only on $n, \gamma$, and $\lambda_{0}$ depends only on $\lambda_{2}, \lambda_{3}$.

For later applications we will give another condition instead of (H2).

Theorem 2.2. Let $V, \lambda_{1}$ and $l$ be as in Theorem 2.1. Suppose that the condition (H1) and the following condition (H2)' hold.

$(\mathrm{H} 2)^{\prime}$ There exist a positive constant $\lambda_{3} \geq \lambda_{1}$ and a function $a_{2}:\left(\lambda_{3}\right.$, $\infty) \rightarrow(0,1 / 2)$ such that

$$
\tilde{G}_{n}(V, \lambda) \leq \lambda^{q_{n}} \sigma(\lambda) a_{2}(\lambda)
$$

for all $\lambda>\lambda_{3}$ where

$$
\tilde{G}_{n}(V, \lambda)=l(\lambda)^{n\left(1-q_{n} / p_{n}\right)} \sum_{\zeta \in I_{\lambda}}\left(\int_{Q_{\zeta}}\left|V(x)-V_{Q_{\zeta}}\right|^{p_{n}} d x\right)^{q_{n} / p_{n}},
$$

$p_{n}=n / 2(n \geq 3), p_{2}=2, p_{1}=1, q_{n}=n / 2(n \geq 2), q_{1}=1 / 4$ and

$$
V_{Q_{\zeta}}=\frac{1}{\left|Q_{\zeta}\right|} \int_{Q_{\zeta}} V(y) d y
$$

Then the same conclusion as in Theorem 2.1 holds.

The following theorem will be used in Section 4 .

Theorem 2.3. Let $V$ and $l$ be as in Theorem 2.1. Suppose that the following conditions hold.

(C1) There exists a positive constant $c_{1}$ such that $\sigma(2 \lambda) \leq c_{1} \sigma(\lambda)$ for large $\lambda$.

(C2) $l(\lambda)^{-1}=o\left(\lambda^{1 / 2}\right)$ as $\lambda \rightarrow \infty$.

(C3) $\widetilde{G}_{n}(V, \lambda)=o\left(\lambda^{q_{n}} \sigma(\lambda)\right)$ where $\widetilde{G}_{n}(V, \lambda)$ is the quantity defined in Theorem 2.2 .

(C4) When $n \geq 3$, we assume

$$
\max _{\zeta \in J \lambda \backslash I_{\lambda}} \operatorname{ess} \sup _{x \in Q_{\zeta}}(\lambda-V(x))_{+}=o(\lambda)
$$

When $n=1$ or 2 , we assume 


$$
\max _{\zeta \in J_{\lambda} \backslash I_{\lambda}} \operatorname{ess} \sup _{x \in Q_{\zeta}}|\lambda-V(x)|=o(\lambda)
$$

Then we have

$$
N(\lambda) \sim(2 \pi)^{-n} \omega_{n} \int_{R^{n}}(\lambda-V)_{+}^{n / 2} d x
$$

as $\lambda \rightarrow \infty$.

\section{$\S 3 . \quad$ Proofs of Theorems}

We shall explain the formulation of eigenvalue problems which is a modification of that of Edmunds and Evans [2]. Let $\Omega$ be a non empty open set in $\boldsymbol{R}^{n}$. Let $W \in L_{\mathrm{loc}}^{1}(\bar{\Omega}), \quad W \geq 1, \quad r \in L^{\infty}(\bar{\Omega})$, and $r \geq 0$. Let $S_{0}(\Omega)=C_{0}^{\infty}(\Omega)$ and $S_{1}(\Omega)$ be the set of all functions on $\Omega$ which are restrictions to $\Omega$ of functions in $C_{0}^{\infty}\left(\boldsymbol{R}^{n}\right)$. Let $H_{i}(W, \Omega)$ be the closure of $S_{i}(\Omega)$, for $i=$ 0,1 , with respect to the norm $(u, u)_{W, \Omega}^{1 / 2}$ where $(u, v)_{W, \Omega}=\int_{\Omega}\{\nabla u \overline{\nabla v}+W u \bar{v}\} d x$, then $H_{i}(W, \Omega)$ is a Hilbert space with the inner product $(\cdot,)_{W, \Omega}$. When $\Omega=\boldsymbol{R}^{n}$, we have $H_{0}\left(W, \boldsymbol{R}^{n}\right)=H_{1}\left(W, \boldsymbol{R}^{n}\right)$ and we denote this space by $H(W)$.

When $\Omega$ is an open cube $Q$ and $W \equiv 1, H_{0}(Q, 1)$ and $H_{1}(Q, 1)$ coincide with usual Sobolev spaces $H_{0}^{1}(Q)$ and $H^{1}(Q)$, respectively (cf. [3, Chapter V, Theorem 4.7]).

We consider the sesquilinear form

$$
a_{i}(u, v)=\int_{\Omega} u \bar{v} r d x
$$

for $u, v \in S_{i}(\Omega)$. Let $H_{i}(W, r, \Omega)$ be the closure of $S_{i}(\Omega)$ with respect to the norm $\left\{(u, u)_{W . \Omega}+a_{i}(u, u)\right\}^{1 / 2}$, then $H_{i}(W, r, \Omega)$ is a Hilbert space with the inner product $(\cdot,)_{W, \Omega}+a_{i}(\cdot, \cdot)$. We can easily show that $a_{i}$ is closable in $H_{i}(W, \Omega)$ and let $\tilde{a}_{i}$ be the closure of $a_{i}$ to $H_{i}(W, r, \Omega)$. Then $\tilde{\mathrm{a}}_{i}$ is a closed, non negative, and symmetric sesquilinear form in $H_{i}(W, \Omega)$ with the domain $H_{i}(W, r, \Omega)$. By the representation theorem, there exists a bounded self-adjoint operator $U_{i}$ (or $U_{i}(W, r, \Omega)$ ) in $H_{i}(W, \Omega)$ such that

$$
\tilde{a}_{i}(u, v)=\left(U_{i} u, v\right)_{W, \Omega}
$$


for all $u, v \in H_{i}(W, \Omega)$.

Let $E_{\eta}\left(U_{i}\right)$ be the resolution of identity corresponding to $U_{i}$. For $\mu>0$, we define

$$
n_{i}(\mu ; W, r, \Omega)=\operatorname{rank} \int_{\mu}^{\infty} d E_{\eta}\left(U_{i}\right)
$$

Here the following three lemmas hold.

Lemma 3.1. Let $\Omega, \Omega_{1}$ and $\Omega_{2}$ be non empty open sets in $\mathbb{R}^{n}$ such that $\Omega_{1} \cap \Omega_{2}=\varnothing, \overline{\Omega_{1} \cup \Omega_{2}}$ has interior equal to $\Omega$ and $\Omega \backslash\left(\Omega_{1} \cup \Omega_{2}\right)$ has Lebesgue n-measure 0. Let $W \in L_{\mathrm{loc}}^{1}(\bar{\Omega}), W \geq 1, r \in L^{\infty}(\bar{\Omega})$, and $r \geq 0$. Then we have

$$
\begin{aligned}
& n_{0}\left(\mu ; W, r, \Omega_{1}\right)+n_{0}\left(\mu ; W, r, \Omega_{2}\right) \leq n_{0}(\mu ; W, r, \Omega) \\
& \leq n_{1}(\mu ; W, r, \Omega) \leq n_{1}\left(\mu ; W, r, \Omega_{1}\right)+n_{1}\left(\mu ; W, r, \Omega_{2}\right)
\end{aligned}
$$

for all $\mu>0$.

Lemma 3.2. Let $\Omega$ be a non empty open set in $R^{n}$ and $W \in L_{\mathrm{loc}}^{1}(\bar{\Omega})$, $W \geq 1$. Let $r_{1}, r_{2} \in L^{\infty}(\bar{\Omega})$ and $0 \leq r_{1}(x) \leq r_{2}(x)$ for a.e. $x \in \bar{\Omega}$. Then

$$
n_{i}\left(\mu ; W, r_{1}, \Omega\right) \leq n_{i}\left(\mu ; W, r_{2}, \Omega\right)
$$

for all $\mu>0$ and $i=0,1$.

Lemma $3.3[1, \mathrm{p} 16,(1.31)]$. Let $\Omega$ be a non empty open set in $\boldsymbol{R}^{n}$ and $W \in L_{\mathrm{loc}}^{1}(\bar{\Omega}), W \geq 1$. Let $r_{1}, r_{2} \in L^{\infty}(\bar{\Omega})$ and $r_{1}, r_{2} \geq 0$. Suppose that $U_{i}(W$, $\left.r_{k}, \Omega\right)$ is a compact operator in $H_{i}(W, \Omega)$ for $i=0,1$ and $k=1,2$. Then

$$
n_{i}\left(\mu_{1}+\mu_{2} ; W, r_{1}+r_{2}, \Omega\right) \leq n_{i}\left(\mu_{1} ; W, r_{1}, \Omega\right)+n_{i}\left(\mu_{2} ; W, r_{2}, \Omega\right),
$$

for all $\mu_{1}, \mu_{2}>0$ and $i=0,1$.

Lemma 3.1 is the formulation of the Dirichlet-Neumann bracketing method which will be used in the present paper. The proofs of Lemmas 3.1 and 3.2 are simple modifications of results in Edmunds and Evans [3, Chapter XI, Section 2.2] and we use the "min-max principle" instead of the 
max-min principle in [3, Chapter XI, Theorem 1.2].

When $\Omega$ is an open cube $Q$ and $W \in L^{\infty}(\bar{Q}), W \geq 1$, we can easily prove that $U_{i}(W, 1, Q)$ is a compact operator in $H_{i}(W, Q)$ for $i=0,1$. Also when $W \equiv 1, r \in L^{\infty}(\bar{Q})$ and $r \geq 0, U_{i}(1, r, Q)$ is a compact operator in $H_{i}(1, Q)$ for $i=0,1$ (cf. [1, p76]).

Throughout the proof, $c$ denotes various positive constants depending only on $n$ and this constant may differ even in the same string of estimates.

Proof of Theorem 2.1. Since $V(x) \rightarrow \infty$ as $|x| \rightarrow \infty$ and $V \geq 1, T$ has the compact inverse operator $T^{-1}$ in $L^{2}\left(\boldsymbol{R}^{n}\right)$ and $T$ has only discrete spectrum ([3, Chapter IV Theorem 2.9, Chapter VIII Theorem 4.1]). Since $S_{0}\left(\boldsymbol{R}^{n}\right)=S_{1}\left(\boldsymbol{R}^{n}\right)=C_{0}^{\infty}\left(\boldsymbol{R}^{n}\right)$, we have $U_{0}\left(V, 1, \boldsymbol{R}^{n}\right)=U_{1}\left(V, 1, \boldsymbol{R}^{n}\right)$. By the definition, $U_{0}\left(V, 1, \boldsymbol{R}^{n}\right)$ is the restriction of $T^{-1}$ to $H(V)$.

Since $T^{-1}$ is a compact operator in $L^{2}\left(R^{n}\right)$, we have, for all $\lambda>0$,

$$
N(\lambda)=\inf _{\mathscr{L} \in K(\lambda)} \operatorname{codim} \mathscr{L}<\infty
$$

where $K(\lambda)$ denotes the set of all subspaces $\mathscr{L} \subset H(V)$ such that

$$
\lambda^{-1} \int_{\boldsymbol{R}^{n}}\left\{|\nabla u|^{2}+V|u|^{2}\right\} d x \geq \int_{\boldsymbol{R}^{n}}|u|^{2} d x
$$

for all $u \in \mathscr{L}$ (cf. [1, p14 Lemma 1.14]). This fact means that $U_{0}\left(V, 1, \boldsymbol{R}^{n}\right)$ is a compact operator in $H(V)$ and

$$
N(\lambda)=n_{0}\left(\lambda^{-1} ; V, 1, \boldsymbol{R}^{n}\right)=n_{1}\left(\lambda^{-1} ; V, 1, \boldsymbol{R}^{n}\right)
$$

for all $\lambda>0$.

We shall estimate $n_{0}\left(\lambda^{-1} ; V, 1, \boldsymbol{R}^{n}\right)$. The arguments of the proof are similar to those of the proof of Theorem 2 in [7]. Let $\lambda_{0}=\max \left\{1, \lambda_{2}, \lambda_{3}\right\}$ and $\lambda>\lambda_{0}$. For simplicity we put $l \equiv l(\lambda)$.

Using Lemma 3.1 and (3.1), we have

$$
\begin{gathered}
\sum_{\zeta \in I_{\lambda}} n_{0}\left(\lambda^{-1} ; V, 1, Q_{\zeta}\right) \leq N(\lambda) \\
\leq \sum_{\zeta \in I_{\lambda}} n_{1}\left(\lambda^{-1} ; V, 1, Q_{\zeta}\right)+\sum_{\zeta \in J_{\lambda} \backslash I_{\lambda}} n_{1}\left(\lambda^{-1} ; V, 1, Q_{\zeta}\right) .
\end{gathered}
$$


In the last inequality, we used the fact that $V(x) \geq \lambda$ for a.e. $x \in \mathbb{R}^{n} \backslash \bigcup_{\zeta \in J_{\lambda}} \overline{Q_{\zeta}}$. Here we set

$$
\varphi(\lambda)=(2 \pi)^{-n} \omega_{n} \int_{\mathbb{R}^{n}}(\lambda-V)_{+}^{n / 2} d x
$$

and we have

$$
\begin{aligned}
& \sum_{\zeta \in I_{\lambda}} n_{0}\left(\lambda^{-1} ; V, 1, Q_{\zeta}\right)-\varphi(\lambda) \leq N(\lambda)-\varphi(\lambda) \\
\leq & \sum_{\zeta \in I_{\lambda}} n_{1}\left(\lambda^{-1} ; V, 1, Q_{\zeta}\right)-\varphi(\lambda)+\sum_{\zeta \in J_{\lambda} \backslash I_{\lambda}} n_{1}\left(\lambda^{-1} ; V, 1, Q_{\zeta}\right) .
\end{aligned}
$$

We set, for $\zeta \in I_{\lambda}$,

$$
\begin{gathered}
r(x)=1-\frac{V(x)}{\lambda}+\frac{1}{\lambda}, \\
r_{\zeta}=\left(\frac{1}{\left|Q_{\zeta}\right|} \int_{Q_{\zeta}}\left(r(x)-\frac{1}{\lambda}\right)^{n / 2} d x\right)^{2 / n}+\frac{1}{\lambda},
\end{gathered}
$$

and

$$
\varphi(\lambda, \zeta)=(2 \pi)^{-n} \omega_{n}\left|Q_{\zeta}\right|\left(\lambda r_{\zeta}-1\right)^{n / 2}
$$

We shall now first find a lower bound for $N(\lambda)-\varphi(\lambda)$ by use of the left-hand side of (3.2); then we shall obtain an analogous upper bound for $N(\lambda)-\varphi(\lambda)$ by means of the right-hand side of (3.2).

Step 1: a lower bound.

For $\zeta \in I_{\lambda}$, the min-max principle gives

$$
n_{i}\left(\lambda^{-1} ; V, 1, Q_{\zeta}\right)=n_{i}\left(\lambda^{-1} ; 1,1-\frac{V}{\lambda}+\frac{1}{\lambda}, Q_{\zeta}\right)
$$

and we have 


$$
\begin{gathered}
\sum_{\zeta \in I_{\lambda}} n_{0}\left(\lambda^{-1} ; V, 1, Q_{\zeta}\right)-\varphi(\lambda)=\sum_{\zeta \in I_{\lambda}} n_{0}\left(\lambda^{-1} ; 1, r, Q_{\zeta}\right)-\varphi(\lambda) \\
=A_{1}+A_{2}+A_{3}
\end{gathered}
$$

where

$$
\begin{gathered}
A_{1}=\sum_{\zeta \in I_{\lambda}}\left\{n_{0}\left(\lambda^{-1} ; 1, r_{\zeta}, Q_{\zeta}\right)-\varphi(\lambda, \zeta)\right\}, \\
A_{2}=\sum_{\zeta \in I_{\lambda}} \varphi(\lambda, \zeta)-\varphi(\lambda),
\end{gathered}
$$

and

$$
A_{3}=\sum_{\zeta \in I_{\lambda}}\left\{n_{0}\left(\lambda^{-1} ; 1, r, Q_{\zeta}\right)-n_{0}\left(\lambda^{-1} ; 1, r_{\zeta}, Q_{\zeta}\right)\right\}
$$

Now we use the following lemma in Edmunds and Evans [3, Chapter XI, Theorem 2.6].

Lemma 3.4. Let $Q$ be an open cube in $\boldsymbol{R}^{n}$ whose sides are parallel to the coordinate axes. Let $\Delta_{0}$ and $\Delta_{1}$ be the Dirichlet and Neumann Laplacian on $Q$, respectively. For $v>0$, let $M_{i}(v)$ denotes the number of eigenvalues of $-\Delta_{i}$ less than $v$. Then there exists a positive constant $c$ depending only on $n$ such that

$$
\left|M_{i}(v)-(2 \pi)^{-n} \omega_{n} v^{n / 2}\right| Q|| \leq c\left\{1+v^{(n-1) / 2}|Q|^{(n-1) / n}\right\}
$$

for all $v>0, i=0,1$.

For all $v>0$ and $i=0,1$, we have $M_{i}(v)=n_{i}\left((v+1)^{-1} ; 1,1, Q\right)$. Hence, by Lemma 3.4, there exists a positive constant $c$ depending only on $n$ such that

$$
\begin{gathered}
\left|n_{i}\left(\mu ; 1,1, Q_{\zeta}\right)-(2 \pi)^{-n} \omega_{n}\left(\mu^{-1}-1\right)^{n / 2}\right| Q_{\zeta}|| \\
\leq c\left\{1+l^{n-1}\left(\mu^{-1}-1\right)^{(n-1) / 2}\right\}
\end{gathered}
$$


for all $\mu$ satisfying $1 \geq \mu>0$. Since

$$
n_{i}\left(\mu\left(r_{\zeta}\right)^{-1} ; 1,1, Q_{\zeta}\right)=n_{i}\left(\mu ; 1, r_{\zeta}, Q_{\zeta}\right)
$$

for $\zeta \in I_{\lambda}$, we have, by (3.4),

$$
\left|n_{i}\left(\mu ; 1, r_{\zeta}, Q_{\zeta}\right)-(2 \pi)^{-n} \omega_{n}\left(r_{\zeta} \mu^{-1}-1\right)^{n / 2}\right| Q_{\zeta} \mid
$$

$$
\leq c\left\{1+l^{n-1}\left(r_{\zeta} \mu^{-1}-1\right)^{(n-1) / 2}\right\}
$$

for all $\mu \leq r_{\zeta}$.

Here we shall estimate $A_{1}$. By (3.5), we have

$$
\left|n_{0}\left(\lambda^{-1} ; 1, r_{\zeta}, Q_{\zeta}\right)-\varphi(\lambda, \zeta)\right| \leq c\left(1+l^{n-1} \lambda^{(n-1) / 2}\right)
$$

We can easily prove that

$$
\sum_{\zeta \in I_{\lambda}}\left|Q_{\zeta}\right| \leq \sigma(\lambda)
$$

and we have

$$
\left|A_{1}\right| \leq c \sigma(\lambda)\left(l^{-n}+\lambda^{(n-1) / 2} l^{-1}\right) .
$$

Next we shall estimate $A_{2}$. We have

$$
\begin{aligned}
& -A_{2}=\varphi(\lambda)-\sum_{\zeta \in I_{\lambda}} \varphi(\lambda, \zeta) \\
& =c \sum_{\zeta \in J_{\lambda} \backslash I_{\lambda}} \int_{Q_{\zeta}}(\lambda-V)_{+}^{n / 2} d x \leq c \lambda^{n / 2}\left(\#\left(J_{\lambda} \backslash I_{\lambda}\right)\right) l^{n} .
\end{aligned}
$$

By the assumption (H1),

$$
\left|A_{2}\right| \leq c \sigma(\lambda) \lambda^{n / 2} a_{1}(\lambda) .
$$

We shall estimate $A_{3}$. 
Let $b(\lambda)=a_{2}(\lambda)^{1 / n}$, then we have, for $\zeta \in I_{\lambda}$,

$$
\begin{gathered}
n_{0}\left(\lambda^{-1} ; 1, r, Q_{\zeta}\right) \leq n_{0}\left(\lambda^{-1} ; 1, r_{\zeta}+\left|r-r_{\zeta}\right|, Q_{\zeta}\right) \\
\leq n_{0}\left((1-b(\lambda)) \lambda^{-1} ; 1, r_{\zeta}, Q_{\zeta}\right)+n_{0}\left(b(\lambda) \lambda^{-1} ; 1,\left|r-r_{\zeta}\right|, Q_{\zeta}\right)
\end{gathered}
$$

where we used Lemmas 3.2 and 3.3 and the fact

$$
b(\lambda)<2^{-1 / n}<1
$$

(3.9) is the consequence of the condition (H2), that is, $a_{2}(\lambda)<1 / 2$.

As a result we have

$$
\begin{aligned}
& \quad n_{0}\left(\lambda^{-1} ; 1, r, Q_{\zeta}\right)-n_{0}\left(\lambda^{-1} ; 1, r_{\zeta}, Q_{\zeta}\right) \\
& \leq n_{0}\left((1-b(\lambda)) \lambda^{-1} ; 1, r_{\zeta}, Q_{\zeta}\right)-n_{0}\left(\lambda^{-1} ; 1, r_{\zeta}, Q_{\zeta}\right) \\
& +n_{0}\left(b(\lambda) \lambda^{-1} ; 1,\left|r-r_{\zeta}\right|, Q_{\zeta}\right) .
\end{aligned}
$$

Similarly

$$
\begin{aligned}
& n_{0}\left(\lambda^{-1} ; 1, r_{\zeta}, Q_{\zeta}\right)-n_{0}\left(\lambda^{-1} ; 1, r, Q_{\zeta}\right) \\
& \leq n_{0}\left(\lambda^{-1} ; 1, r_{\zeta}, Q_{\zeta}\right)-n_{0}\left((1+b(\lambda)) \lambda^{-1} ; 1, r_{\zeta}, Q_{\zeta}\right) \\
& +n_{0}\left(b(\lambda) \lambda^{-1} ; 1,\left|r-r_{\zeta}\right|, Q_{\zeta}\right) .
\end{aligned}
$$

We shall estimate (3.10). By (3.5) and (3.9), we have

$$
\begin{gathered}
n_{0}\left((1-b(\lambda)) \lambda^{-1} ; 1, r_{\zeta}, Q_{\zeta}\right)-n_{0}\left(\lambda^{-1} ; 1, r_{\zeta}, Q_{\zeta}\right) \\
\leq c\left\{\left(\lambda r_{\zeta}(1-b(\lambda))^{-1}-1\right)^{n / 2}-\left(\lambda r_{\zeta}-1\right)^{n / 2}\right\}\left|Q_{\zeta}\right|+c+c \lambda^{(n-1) / 2}\left|Q_{\zeta}\right| l^{-1} .
\end{gathered}
$$

If $n \geq 2$, then we have

$$
\begin{gathered}
\left(\lambda r_{\zeta}(1-b(\lambda))^{-1}-1\right)^{n / 2}-\left(\lambda r_{\zeta}-1\right)^{n / 2} \\
\leq c\left(\lambda r_{\zeta}(1-b(\lambda))^{-1}-1\right)^{n / 2-1} \lambda r_{\zeta} b(\lambda)(1-b(\lambda))^{-1} .
\end{gathered}
$$

If $n=1$, then we have 


$$
\left(\lambda r_{\zeta}(1-b(\lambda))^{-1}-1\right)^{1 / 2}-\left(\lambda r_{\zeta}-1\right)^{1 / 2} \leq\left\{\lambda r_{\zeta} b(\lambda)(1-b(\lambda))^{-1}\right\}^{1 / 2}
$$

Therefore

$$
\begin{aligned}
& n_{0}\left((1-b(\lambda)) \lambda^{-1} ; 1, r_{\zeta}, Q_{\zeta}\right)-n_{0}\left(\lambda^{-1} ; 1, r_{\zeta}, Q_{\zeta}\right) \\
& \quad \leq c+c a_{2}(\lambda)^{\theta_{n}} \lambda^{n / 2}\left|Q_{\zeta}\right|+c \lambda^{(n-1) / 2}\left|Q_{\zeta}\right| l^{-1}
\end{aligned}
$$

where $\theta_{n}=\min \{1 / 2,1 / n\}$.

Next we estimate $n_{0}\left(b(\lambda) \lambda^{-1} ; 1,\left|r-r_{\zeta}\right|, Q_{\zeta}\right)$ by means of the following lemma ([1, p.80, Remark 4.3]).

Lemma 3.5. Let $Q$ be an open cube in $\mathbb{R}^{n}$ whose sides are parallel to the coordinate axes and let $\rho \in L^{\infty}(Q), \rho \geq 0$. Then there exists a positive constant $c$ depending only on $n$ such that

$$
n_{i}(\mu ; 1, \rho, Q) \leq c \mu^{-n / 2}\|\rho\|_{p_{n}, Q}^{n / 2}|Q|^{1-n /\left(2 p_{n}\right)}
$$

for all $\mu>0, i=0,1$, where $p_{n}$ is the number defined in the condition (H2).

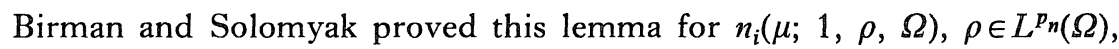
and their estimate for $n_{1}(\mu ; 1, \rho, \Omega)$ is not the same form as that in Lemma 3.5 When $\Omega$ is a cube $Q$, the precise calculation in their proof leads is our estimate.

Using Lemma 3.5 and the definition of $b(\lambda)$, we have

$$
\begin{aligned}
& n_{0}\left(b(\lambda) \lambda^{-1} ; 1,\left|r-r_{\zeta}\right|, Q_{\zeta}\right) \\
& \quad \leq c a_{2}(\lambda)^{-1 / 2} \lambda^{n / 2}\left\|r-r_{\zeta}\right\|_{p_{n}, Q_{\zeta}}^{n / 2}\left|Q_{\zeta}\right|^{1-n /\left(2 p_{n}\right)} .
\end{aligned}
$$

Hence, by (3.10), (3.12) and (3.13),

$$
\begin{aligned}
& n_{0}\left(\lambda^{-1} ; 1, r, Q_{\zeta}\right)-n_{0}\left(\lambda^{-1} ; 1, r_{\zeta}, Q_{\zeta}\right) \\
& \leq c+c a_{2}(\lambda)^{\theta_{n}} \lambda^{n / 2}\left|Q_{\zeta}\right|+c \lambda^{(n-1) / 2}\left|Q_{\zeta}\right| l^{-1} \\
& +c a_{2}(\lambda)^{-1 / 2} \lambda^{n / 2}\left\|r-r_{\zeta}\right\|_{p_{n}, Q_{\zeta}}^{n / 2}\left|Q_{\zeta}\right|^{1-n /\left(2 p_{n}\right)} .
\end{aligned}
$$

Similarly we can estimate (3.11). If $\lambda r_{\zeta} \geq 1+b(\lambda)$, that is, 


$$
b(\lambda) \leq\left(\frac{1}{\left|Q_{\zeta}\right|} \int_{Q_{\zeta}}(\lambda-V)^{n / 2} d x\right)^{2 / n},
$$

then similar arguments as in (3.12) lead to the estimate

$$
\begin{gathered}
n_{0}\left(\lambda^{-1} ; 1, r_{\zeta}, Q_{\zeta}\right)-n_{0}\left((1+b(\lambda)) \lambda^{-1} ; 1, r_{\zeta}, Q_{\zeta}\right) \\
\leq c+c a_{2}(\lambda)^{\theta_{n}} \lambda^{n / 2}\left|Q_{\zeta}\right|+c \lambda^{(n-1) / 2}\left|Q_{\zeta}\right| l^{-1} .
\end{gathered}
$$

If $\lambda r_{\zeta}<1+b(\lambda)$, that is,

$$
b(\lambda)>\left(\frac{1}{\left|Q_{\zeta}\right|} \int_{Q_{\zeta}}(\lambda-V)^{n / 2} d x\right)^{2 / n},
$$

then we have $n_{0}\left((1+b(\lambda)) \lambda^{-1} ; 1, r_{\zeta}, Q_{\zeta}\right)=0$ and

$$
\begin{aligned}
n_{0}\left(\lambda^{-1} ; 1, r_{\zeta}, Q_{\zeta}\right) & \leq c+c\left(\lambda r_{\zeta}-1\right)^{n / 2}\left|Q_{\zeta}\right|+c \lambda^{(n-1) / 2}\left|Q_{\zeta}\right| l^{-1} \\
& \leq c+c b(\lambda)^{n / 2}\left|Q_{\zeta}\right|+c \lambda^{(n-1) / 2}\left|Q_{\zeta}\right| l^{-1} \\
& \leq c+c a_{2}(\lambda)^{\theta_{n}}\left|Q_{\zeta}\right|+c \lambda^{(n-1) / 2}\left|Q_{\zeta}\right| l^{-1}
\end{aligned}
$$

where we used the fact $a_{2}(\lambda)^{1 / 2} \leq a_{2}(\lambda)^{\theta_{n}}$.

Therefore we have, by (3.11) and (3.13)

$$
\begin{aligned}
n_{0}\left(\lambda^{-1} ; 1, r_{\zeta}, Q_{\zeta}\right)-n_{0}\left(\lambda^{-1} ; 1, r, Q_{\zeta}\right) \\
\leq c+c a_{2}(\lambda)^{\theta_{n}} \lambda^{n / 2}\left|Q_{\zeta}\right|+c \lambda^{(n-1) / 2}\left|Q_{\zeta}\right| l^{-1} \\
\quad+c a_{2}(\lambda)^{-1 / 2} \lambda^{n / 2}\left\|r-r_{\zeta}\right\|_{p_{n}, Q_{\zeta}}^{n / 2}\left|Q_{\zeta}\right|^{1-n /\left(2 p_{n}\right)}
\end{aligned}
$$

Accordingly, by (3.14), (3.15), and the definitions of $g$ and $g_{\zeta}$,

$$
\begin{aligned}
\left|A_{3}\right| & \leq \sum_{\zeta \in I_{\lambda}}\left|n_{0}\left(\lambda^{-1} ; 1, r, Q_{\zeta}\right)-n_{0}\left(\lambda^{-1} ; 1, r_{\zeta}, Q_{\zeta}\right)\right| \\
& \leq c\left(\# I_{\lambda}\right)+c a_{2}(\lambda)^{\theta_{n}} \lambda^{n / 2} \sigma(\lambda)+c \lambda^{(n-1) / 2} l^{-1} \sigma(\lambda) \\
& +c a_{2}(\lambda)^{-1 / 2} \lambda^{n / 2} \sum_{\zeta \in I_{\lambda}}\left\|g-g_{\zeta}\right\|_{p_{n}, Q_{\zeta}}^{n / 2}\left|Q_{\zeta}\right|^{1-n /\left(2 p_{n}\right)} .
\end{aligned}
$$

By the assumption ( $\mathrm{H} 2)$, we have 


$$
c a_{2}(\lambda)^{-1 / 2} \lambda^{n / 2} \sum_{\zeta \in I_{\lambda}}\left\|g-g_{\zeta}\right\|_{p_{n}, Q_{\zeta}}^{n / 2}\left|Q_{\zeta}\right|^{1-n /\left(2 p_{n}\right)} \leq c \gamma \lambda^{n / 2} \sigma(\lambda) a_{2}(\lambda)^{1 / 2} .
$$

\section{Hence}

$$
\left|A_{3}\right| \leq c(1+\gamma) \sigma(\lambda)\left\{\lambda^{n / 2} a_{2}(\lambda)^{\theta_{n}}+l^{-n}+\lambda^{(n-1) / 2} l^{-1}\right\} .
$$

By (3.2), (3.3), (3.6), (3.8), and (3.16),

$$
\begin{aligned}
& N(\lambda)-\varphi(\lambda) \geq-\left|A_{1}\right|-\left|A_{2}\right|-\left|A_{3}\right| \\
\geq & -c(1+\gamma) \sigma(\lambda)\left\{\lambda^{n / 2} a_{1}(\lambda)+\lambda^{n / 2} a_{2}(\lambda)^{\theta_{n}}+l^{-n}+\lambda^{(n-1) / 2} l^{-1}\right\} .
\end{aligned}
$$

This completes Step 1.

Step 2: an upper bound.

We have

$$
\begin{gathered}
\sum_{\zeta \in I_{\lambda}} n_{1}\left(\lambda^{-1} ; V, 1, Q_{\zeta}\right)-\varphi(\lambda)+\sum_{\zeta \in J_{\lambda} \backslash I_{\lambda}} n_{1}\left(\lambda^{-1} ; V, 1, Q_{\zeta}\right) \\
=A_{1}^{\prime}+A_{2}^{\prime}+A_{3}^{\prime}+A_{4}^{\prime}
\end{gathered}
$$

where

$$
\begin{gathered}
A_{1}^{\prime}=\sum_{\zeta \in I_{\lambda}}\left\{n_{1}\left(\lambda^{-1} ; 1, r_{\zeta}, Q_{\zeta}\right)-\varphi(\lambda, \zeta)\right\}, \\
A_{2}^{\prime}=\sum_{\zeta \in I_{\lambda}} \varphi(\lambda, \zeta)-\varphi(\lambda), \\
A_{3}^{\prime}=\sum_{\zeta \in I_{\lambda}}\left\{n_{1}\left(\lambda^{-1} ; 1, r, Q_{\zeta}\right)-n_{1}\left(\lambda^{-1} ; 1, r_{\zeta}, Q_{\zeta}\right)\right\},
\end{gathered}
$$

and

$$
A_{4}^{\prime}=\sum_{\zeta \in J_{\lambda} \backslash I_{\lambda}} n_{1}\left(\lambda^{-1} ; V, 1, Q_{\zeta}\right)
$$

We can estimate $A_{1}^{\prime}, A_{2}^{\prime}$ and $A_{3}^{\prime}$ as in Step 1 , that is, we have 


$$
\left|A_{1}^{\prime}\right|+\left|A_{2}^{\prime}\right|+\left|A_{3}^{\prime}\right|
$$

$$
\leq c(1+\gamma) \sigma(\lambda)\left\{\lambda^{n / 2} a_{1}(\lambda)+\lambda^{n / 2} a_{2}(\lambda)^{\theta_{n}}+l^{-n}+\lambda^{(n-1) / 2} l^{-1}\right\}
$$

We shall estimate $A_{4}^{\prime}$. Since $V \geq 1$, we have, by the min-max principle,

$$
A_{4}^{\prime}=\sum_{\zeta \in J_{\lambda} \backslash I_{\lambda}} n_{1}\left(\lambda^{-1} ; V, 1, Q_{\zeta}\right) \leq \sum_{\zeta \in J_{\lambda} \backslash I_{\lambda}} n_{1}\left(\lambda^{-1} ; 1,1, Q_{\zeta}\right)
$$

By (3.4) the last term does not exceed

$$
\left(c \lambda^{n / 2} l^{n}+c+c \lambda^{(n-1) / 2} l^{n-1}\right)\left(\#\left(J_{\lambda} \backslash I_{\lambda}\right)\right) .
$$

By means of the assumption (H1), we have

$$
A_{4}^{\prime} \leq c \lambda^{n / 2} \sigma(\lambda) a_{1}(\lambda)+c \sigma(\lambda) l^{-n}+c \lambda^{(n-1) / 2} \sigma(\lambda) l^{-1}
$$

where we used the fact $a_{1}(\lambda) \leq 1$.

By (3.2), (3.18), (3.19), and (3.20),

$$
N(\lambda)-\varphi(\lambda)
$$

$$
\leq c(1+\gamma) \sigma(\lambda)\left\{\lambda^{n / 2} a_{1}(\lambda)+\lambda^{n / 2} a_{2}(\lambda)^{\theta_{n}}+l^{-n}+\lambda^{(n-1) / 2} l^{-1}\right\}
$$

This completes Step 2.

By (3.17) and (3.21), we have

$$
\begin{aligned}
& \left|N(\lambda)-(2 \pi)^{-n} \omega_{n} \int_{R^{n}}(\lambda-V)_{+}^{n / 2} d x\right| \\
& \leq c(1+\gamma) \sigma(\lambda)\left\{\lambda^{n / 2} a_{1}(\lambda)+\lambda^{n / 2} a_{2}(\lambda)^{\theta_{n}}+l(\lambda)^{-n}+\lambda^{(n-1) / 2} l(\lambda)^{-1}\right\}
\end{aligned}
$$

for all $\lambda>\lambda_{0}$.

Q.E.D.

\section{Proof of Theorem 2.2.}

First we consider the case $n \geq 2$. We will establish the inequality

$$
\left(\int_{Q_{\zeta}}\left|g-g_{\zeta}\right|^{p_{n}} d x\right)^{n /\left(2 p_{n}\right)} \leq \gamma \lambda^{-n / 2}\left(\int_{Q_{\zeta}}\left|V-V_{Q_{\zeta}}\right|^{p_{n}} d x\right)^{n /\left(2 p_{n}\right)}
$$


for all $\zeta \in I_{\lambda}$ and all $\lambda>\lambda_{3}$ where $\gamma$ is a positive constant depending only on $n$. If (3.22) holds, then we get Theorem 2.2 by Theorem 2.1 .

When $n=2$, we have

$$
\left(\int_{Q_{\zeta}}\left|g-g_{\zeta}\right|^{2} d x\right)^{1 / 2}=\lambda^{-1}\left(\int_{Q_{\zeta}}\left|V-V_{Q_{\zeta}}\right|^{2} d x\right)^{1 / 2}
$$

Hence (3.22) holds.

When $n \geq 3$, we have, for $\zeta \in I_{\lambda}$,

$$
\lambda^{-n / 2} \int_{Q_{\zeta}}\left|V-V_{Q_{\zeta}}\right|^{n / 2} d x=\int_{Q_{\zeta}}\left|g-g_{Q_{\zeta}}\right|^{n / 2} d x
$$

where

$$
g_{Q_{\zeta}}=\frac{1}{\left|Q_{\zeta}\right|} \int_{Q_{\zeta}} g(y) d y=\frac{1}{\left|Q_{\zeta}\right|} \int_{Q_{\zeta}}\left(1-\frac{V(y)}{\lambda}\right) d y
$$

We shall prove the inequality

$$
\int_{Q_{\zeta}}\left|g-g_{\zeta}\right|^{n / 2} d x \leq \gamma \int_{Q_{\zeta}}\left|g-g_{Q_{\zeta}}\right|^{n / 2} d x
$$

for some positive constant $\gamma$ depending only on $n$.

By Minkowski's inequality,

$$
\left(\int_{Q_{\zeta}}\left|g-g_{\zeta}\right|^{n / 2} d x\right)^{2 / n} \leq\left(\int_{Q_{\zeta}}\left|g-g_{Q_{\zeta}}\right|^{n / 2} d x\right)^{2 / n}+\left|g_{\zeta}-g_{Q_{\zeta}}\right| \cdot\left|Q_{\zeta}\right|^{2 / n}
$$

Now

$$
g_{\zeta}=\left(\frac{1}{\left|Q_{\zeta}\right|} \int_{Q_{\zeta}} g^{n / 2} d x\right)^{2 / n} \leq\left(\frac{1}{\left|Q_{\zeta}\right|} \int_{Q_{\zeta}}\left|g-g_{Q_{\zeta}}\right|^{n / 2} d x\right)^{2 / n}+g_{Q_{\zeta}}
$$

Hence

$$
\left|g_{\zeta}-g_{Q_{\zeta}}\right|=g_{\zeta}-g_{Q_{\zeta}} \leq\left(\frac{1}{\left|Q_{\zeta}\right|} \int_{Q_{\zeta}}\left|g-g_{Q_{\zeta}}\right|^{n / 2} d x\right)^{2 / n}
$$


As a result we have

$$
\int_{Q_{\zeta}}\left|g-g_{\zeta}\right|^{n / 2} d x \leq 2^{n / 2} \int_{Q_{\zeta}}\left|g-g_{Q_{\zeta}}\right|^{n / 2} d x
$$

which is (3.24). Hence we have (3.22).

Next we consider the case $n=1$.

We shall prove

$$
\begin{aligned}
G_{1}(V, \lambda) & =l(\lambda)^{1 / 2} \sum_{\zeta \in I_{\lambda}}\left(\int_{Q_{\zeta}}\left|g-g_{\zeta}\right| d x\right)^{1 / 2} \\
& \leq \gamma l(\lambda)^{3 / 4} \sum_{\zeta \in I_{\lambda}}\left(\int_{Q_{\zeta}}\left|g-g_{Q_{\zeta}}\right| d x\right)^{1 / 4}
\end{aligned}
$$

for all $\lambda>\lambda_{3}$ where $\gamma$ is a positive constant not depending on $\lambda$ and $\zeta$. If (3.25) holds, then we have, by (H2)',

$$
G_{1}(V, \lambda) \leq \gamma \sigma(\lambda) a_{2}(\lambda)
$$

which is $(\mathrm{H} 2)$.

(3.25) is the consequence of the inequality

$$
\int_{Q_{\zeta}}\left|g-g_{\zeta}\right| d x \leq \gamma l(\lambda)^{1 / 2}\left(\int_{Q_{\zeta}}\left|g-g_{Q_{\zeta}}\right| d x\right)^{1 / 2}
$$

for all $\zeta \in I_{\lambda}$ and all $\lambda>\lambda_{3}$. We shall prove (3.26). Now

$$
\int_{Q_{\zeta}}\left|g-g_{\zeta}\right| d x \leq \int_{Q_{\zeta}}\left|g-g_{Q_{\zeta}}\right| d x+\left|g_{\zeta}-g_{Q_{\zeta}}\right| \cdot\left|Q_{\zeta}\right|
$$

We shall estimate the first term on the right-hand side of (3.27). By Hölder's inequality, we have

$$
\begin{aligned}
\int_{Q_{\zeta}}\left|g-g_{Q_{\zeta}}\right| d x & \leq\left|Q_{\zeta}\right|^{1 / 2}\left(\int_{Q_{\zeta}}\left|g-g_{Q_{\zeta}}\right|^{2} d x\right)^{1 / 2} \\
& \leq \sqrt{2}\left|Q_{\zeta}\right|^{1 / 2}\left(\int_{Q_{\zeta}}\left|g-g_{Q_{\zeta}}\right| d x\right)^{1 / 2}
\end{aligned}
$$


where we used the fact that $|g(x)| \leq 1$ a.e. $x \in Q_{\zeta}$.

Next we shall estimate the second term on the right-hand side of (3.27). We have

$$
\begin{gathered}
\left|g_{\zeta}-g_{Q_{\zeta}}\right| \leq 2\left|\left(g_{\zeta}\right)^{1 / 2}-\left(g_{Q_{\zeta}}\right)^{1 / 2}\right| \leq \frac{2}{\left|Q_{\zeta}\right|} \int_{Q_{\zeta}}\left|g^{1 / 2}-\left(g_{Q_{\zeta}}\right)^{1 / 2}\right| d x \\
\quad \leq \frac{2}{\left|Q_{\zeta}\right|} \int_{Q_{\zeta}}\left|g-g_{Q_{\zeta}}\right|^{1 / 2} d x \leq 2\left(\frac{1}{\left|Q_{\zeta}\right|} \int_{Q_{\zeta}}\left|g-g_{Q_{\zeta}}\right| d x\right)^{1 / 2} .
\end{gathered}
$$

Hence

$$
\left.\left|g_{\zeta}-g_{Q_{\zeta}} \cdot\right| Q_{\zeta}|\leq 2| Q_{\zeta}\right|^{1 / 2}\left(\int_{Q_{\zeta}}\left|g-g_{Q_{\zeta}}\right| d x\right)^{1 / 2}
$$

By (3.27), (3.28) and (3.29), we have (3.26).

Q.E.D.

Proof of Theorem 2.3. For simplicity we set

$$
\Psi(\lambda)=\int_{R^{n}}(\lambda-V)_{+}^{n / 2} d x
$$

By the condition (C1) we have $\lambda^{n / 2} \sigma(\lambda)=O(\Psi(\lambda)$ ) as $\lambda \rightarrow \infty$. Hence, by (C2), (C3), (2.6) and (2.16), we have

$$
\left|A_{1}\right|+\left|A_{3}\right|+\left|A_{1}^{\prime}\right|+\left|A_{3}^{\prime}\right|=o(\Psi(\lambda))
$$

where $A_{1}, A_{3}, A_{1}^{\prime}, A_{3}^{\prime}$ are quantities in the proof of Theorem 2.1 and we used the condition (H2)' in Theorem 2.2.

We shall estimate $A_{2}, A_{2}^{\prime}$, and $A_{4}^{\prime}$. We set

$$
\varepsilon(\lambda)=\lambda^{-1} \max _{\zeta \in J_{\lambda} \backslash I_{\lambda}} \text { ess } \sup _{x \in Q_{\zeta}}(\lambda-V(x))_{+}
$$

and

$$
\eta(\lambda)=\lambda^{-1} \max _{\zeta \in J_{\lambda} \backslash I_{\lambda}} \operatorname{ess} \sup _{x \in Q_{\zeta}}|\lambda-V(x)|
$$


By (3.7), we have

$$
\left|A_{2}\right|=\left|A_{2}^{\prime}\right|=c \sum_{\zeta \in J_{\lambda} \backslash I_{\lambda}} \int_{Q_{\zeta}}(\lambda-V)_{+}^{n / 2} d x \leq c \varepsilon(\lambda)^{n / 2} \lambda^{n / 2} \sigma(\lambda) .
$$

By the condition (C4), we have

$$
\left|A_{2}\right|=\left|A_{2}^{\prime}\right|=o(\Psi(\lambda))
$$

Next we shall estimate $A_{4}^{\prime}$. Now we have, for $\zeta \in J_{\lambda} \backslash I_{\lambda}$,

$$
n_{1}\left(\lambda^{-1} ; V, 1, Q_{\zeta}\right)=\inf _{\mathscr{L}_{\in} \in K_{\zeta}(\lambda)} \operatorname{codim} \mathscr{L}
$$

where $K_{\zeta}(\lambda)$ denotes the set of all subspaces $\mathscr{L} \subset S_{1}\left(Q_{\zeta}\right)$ such that

$$
\lambda^{-1} \int_{Q_{\zeta}}\left\{|\nabla u|^{2}+V|u|^{2}\right\} d x \geq \int_{Q_{\zeta}}|u|^{2} d x
$$

for all $u \in \mathscr{L}$ and $S_{1}\left(Q_{\zeta}\right)$ is the space defined in the beginning of this section.

If we denote $\tilde{K}_{\zeta}(\lambda)$ the set of all subspaces $\mathscr{L} \subset S_{1}\left(Q_{\zeta}\right)$ such that

$$
\lambda^{-1} \int_{Q_{\zeta}}\left\{|\nabla u|^{2}+|u|^{2}\right\} d x \geq \int_{Q_{\zeta}}\left(1+\frac{1}{\lambda}-\frac{V}{\lambda}\right)_{+}|u|^{2} d x
$$

for all $u \in \mathscr{L}$, then we have $\tilde{K}_{\zeta}(\lambda) \subset K_{\zeta}(\lambda)$ and

$$
n_{1}\left(\lambda^{-1} ; 1,\left(1+\lambda^{-1}-V \lambda^{-1}\right)_{+}, Q_{\zeta}\right)=\inf _{\mathscr{L}_{\widetilde{K}_{\zeta}(\lambda)}} \operatorname{codim} \mathscr{L}
$$

Hence we have

$$
n_{1}\left(\lambda^{-1} ; V, 1, Q_{\zeta}\right) \leq n_{1}\left(\lambda^{-1} ; 1,\left(1+\lambda^{-1}-V \lambda^{-1}\right)_{+}, Q_{\zeta}\right)
$$

By Lemma 3.5, we have

$$
n_{1}\left(\lambda^{-1} ; V, 1, Q_{\zeta}\right) \leq c\left\{\int_{Q_{\zeta}}(\lambda+1-V)_{+}^{p_{n}} d x\right\}^{n /\left(2 p_{n}\right)}\left|Q_{\zeta}\right|^{1-n /\left(2 p_{n}\right)}
$$


As a result

$$
\begin{aligned}
A_{4}^{\prime} & =\sum_{\zeta \in J_{\lambda} \backslash I_{\lambda}} n_{1}\left(\lambda^{-1} ; V, 1, Q_{\zeta}\right) \\
& \leq \sum_{\zeta \in J_{\lambda} \backslash I_{\lambda}} c\left\{\int_{Q_{\zeta}}(\lambda+1-V)^{p_{n}} d x\right\}^{n /\left(2 p_{n}\right)}\left|Q_{\zeta}\right|^{1-n /\left(2 p_{n}\right)}
\end{aligned}
$$

First we consider the case $n \geq 3$. Since $p_{n}=n / 2$, we have

$$
\begin{aligned}
A_{4}^{\prime} & \leq \sum_{\zeta \in J_{\lambda} \backslash I_{\lambda}} c \int_{Q_{\zeta}}(\lambda+1-V)_{+}^{n / 2} d x \\
& \leq c\left\{\int_{R^{n}}(\lambda+1-V)_{+}^{n / 2} d x-\sum_{\zeta \in I_{\lambda}} \int_{Q_{\zeta}}(\lambda+1-V)_{+}^{n / 2} d x\right\} \\
& \leq c\{\Psi(\lambda+1)-\Psi(\lambda)\}+c \sum_{\zeta \in J_{\lambda} \backslash I_{\lambda}} \int_{Q_{\zeta}}(\lambda-V)_{+}^{n / 2} d x \\
& \leq c\{\Psi(\lambda+1)-\Psi(\lambda)\}+c \varepsilon(\lambda)^{n / 2} \lambda^{n / 2} \sigma(\lambda) .
\end{aligned}
$$

By (C2) and Lemma 1.1 in [12], we have

$$
\lim _{\lambda \rightarrow \infty} \frac{\Psi(\lambda+1)}{\Psi(\lambda)}=1
$$

Therefore, by (C4),

$$
A_{4}^{\prime}=o(\Psi(\lambda))
$$

Next we consider the case $n=1$ or 2 . By (3.32), we have

$$
A_{4}^{\prime} \leq(\eta(\lambda) \lambda+1)^{n / 2} \sum_{\zeta \in J_{\lambda} \backslash I_{\lambda}}\left|Q_{\zeta}\right|
$$

Since

$\max$ ess $\sup V(x) \leq \lambda+\eta(\lambda) \lambda<2 \lambda$ 
for large $\lambda$, we have, by (C1),

$$
\sum_{\zeta \in J_{\lambda} \backslash I_{\lambda}}\left|Q_{\zeta}\right| \leq \sigma(2 \lambda) \leq c_{1} \sigma(\lambda)
$$

Hence we have

$$
A_{4}^{\prime}=o(\Psi(\lambda))
$$

By (3.30), (3.31), (3.33) and (3.34), we have

$$
N(\lambda) \sim(2 \pi)^{-n} \omega_{n} \int_{R^{n}}(\lambda-V)_{+}^{n / 2} d x \text { as } \lambda \rightarrow \infty .
$$

Q.E.D.

\section{$\S 4$. Applications}

In this section we will study some applications of our main results in Section 2. First we consider radial potentials.

Theorem 4.1. Let $f \geq 1$ be a strictly increasing function on $[0, \infty)$ such that $f \in C^{1}([0, \infty))$ and $f(t) \rightarrow \infty$ as $t \rightarrow \infty$. We assume, for a positive integer $n$,

$$
\lambda^{n / 2}\left\{f^{-1}(\lambda)\right\}^{n-v_{n}}=o\left(\int_{0}^{f^{-1}(\lambda)}(\lambda-f(t))^{n / 2} t^{n-1} d t\right) \text { as } \lambda \rightarrow \infty
$$

where $v_{n}=1 /(2 n)(n \geq 3), v_{2}=v_{1}=1 / 8$. Let $V(x)=f(|x|)$ for $x \in \boldsymbol{R}^{n}$. Then

$$
N(\lambda) \sim(2 \pi)^{-n} \omega_{n} \int_{R^{n}}(\lambda-V)_{+}^{n / 2} d x \quad \text { as } \lambda \rightarrow \infty
$$

Proof. In Theorem 2.1 we set $l(\lambda)=\left\{f^{-1}(\lambda)\right\}^{1 / 2}$. We shall calculate $a_{1}(\lambda)$ and $a_{2}(\lambda)$ in $(\mathrm{H} 1)$ and $(\mathrm{H} 2)$.

We consider the condition (H1). By a simple geometrical consideration, we have

$$
\frac{\left(\#\left(J_{\lambda} \backslash I_{\lambda}\right)\right)}{\left(\# I_{\lambda}\right)} \leq c\left\{f^{-1}(\lambda)\right\}^{-1 / 2}
$$


for sufficiently large $\lambda$ where $c$ is a positive constant depending only on $n$.

Next we consider the condition (H2). First we consider the case $n \geq 3$. We have

$$
\begin{aligned}
G_{n}(V, \lambda) & =\sum_{\zeta \in I_{\lambda}} \int_{Q_{\zeta}}\left|g-g_{\zeta}\right|^{n / 2} d x \leq \sum_{\zeta \in I_{\lambda}} \int_{Q_{\zeta}}\left|g^{n / 2}-\left(g_{\zeta}\right)^{n / 2}\right| d x \\
& \leq c \sum_{\zeta \in I_{\lambda}} l(\lambda) \int_{Q_{\zeta}}\left|\nabla\left(g^{n / 2}\right)\right| d x \leq c l(\lambda) \int_{\Omega_{\lambda}}\left|\nabla\left(g^{n / 2}\right)\right| d x
\end{aligned}
$$

where $\Omega_{\lambda}=\left\{x \in \boldsymbol{R}^{n}: V(x)<\lambda\right\}$ and we used the Poincaré's inequality ([3, p.242, Theorem 3.23]).

Here we have

$$
\int_{\Omega_{\lambda}}\left|\nabla\left(g^{n / 2}\right)\right| d x=c \int_{0}^{f^{-1}(\lambda)} \frac{n}{2}\left(1-\frac{f(t)}{\lambda}\right)^{n / 2-1} \frac{1}{\lambda} f^{\prime}(t) t^{n-1} d t \leq c\left\{f^{-1}(\lambda)\right\}^{n-1}
$$

Accordingly we have

$$
G_{n}(V, \lambda) \leq c l(\lambda)\left\{f^{-1}(\lambda)\right\}^{n-1}=c \sigma(\lambda)\left\{f^{-1}(\lambda)\right\}^{-1 / 2}
$$

for large $\lambda$.

Secondly we consider the case $n=2$.

$$
G_{2}(V, \lambda)=l(\lambda) \sum_{\zeta \in I_{\lambda}}\left(\int_{Q_{\zeta}}\left|g-g_{\zeta}\right|^{2} d x\right)^{1 / 2} \leq \operatorname{cl}(\lambda) \sum_{\zeta \in I_{\lambda}}\left(\int_{Q_{\zeta}}\left|g-g_{\zeta}\right| d x\right)^{1 / 2}
$$

where we used the fact that $|g| \leq 1$. The last term does not exceed

$$
\begin{aligned}
& c l(\lambda)^{3 / 2}\left(\sum_{\zeta \in I_{\lambda}} \int_{Q_{\zeta}}|\nabla g| d x\right)^{1 / 2}\left(\# I_{\lambda}\right)^{1 / 2} \\
& \quad \leq c \sigma(\lambda)^{1 / 2} l(\lambda)^{1 / 2}\left(\int_{0}^{f^{-1}(\lambda)} \frac{1}{\lambda} f^{\prime}(t) t d t\right)^{1 / 2} \leq c \sigma(\lambda)\left\{f^{-1}(\lambda)\right\}^{-1 / 4} .
\end{aligned}
$$

Hence we have

$$
G_{2}(V, \lambda) \leq c \sigma(\lambda)\left\{f^{-1}(\lambda)\right\}^{-1 / 4}
$$


Finally we consider the case $n=1$.

$$
\begin{aligned}
& G_{1}(V, \lambda)=l(\lambda)^{1 / 2} \sum_{\zeta \in I_{\lambda}}\left(\int_{Q_{\zeta}}\left|g-g_{\zeta}\right| d x\right)^{1 / 2} \\
& \quad \leq c l(\lambda)^{1 / 2} \sum_{\zeta \in I_{\lambda}}\left(\int_{Q_{\zeta}}\left|g^{1 / 2}-\left(g_{\zeta}\right)^{1 / 2}\right| d x\right)^{1 / 2} \\
& \quad \leq c \sigma(\lambda)^{1 / 2}\left(\sum_{\zeta \in I_{\lambda}} \int_{Q_{\zeta}}\left|g^{1 / 2}-\left(g_{\zeta}\right)^{1 / 2}\right| d x\right)^{1 / 2} .
\end{aligned}
$$

As a result we have

$$
\begin{aligned}
G_{1}(V, \lambda) & \leq c \sigma(\lambda)^{1 / 2} l(\lambda)^{1 / 2}\left(\int_{0}^{f^{-1}(\lambda)}\left(1-\frac{f}{\lambda}\right)^{-1 / 2} \frac{f^{\prime}}{\lambda} d t\right)^{1 / 2} \\
& \leq c \sigma(\lambda)^{1 / 2} l(\lambda)^{1 / 2}=c \sigma(\lambda)\left\{f^{-1}(\lambda)\right\}^{-1 / 4}
\end{aligned}
$$

Hence we have

$$
G_{n}(V, \lambda) \leq c \sigma(\lambda)\left\{f^{-1}(\lambda)\right\}^{-\mu_{n}}
$$

for $n \geq 1$ and for large $\lambda$ where $\mu_{n}=1 / 2(n \geq 3), \mu_{2}=\mu_{1}=1 / 4$. By Theorem 2.1, we have

$$
\left|N(\lambda)-(2 \pi)^{-n} \omega_{n} \int_{\boldsymbol{R}^{n}}(\lambda-V)_{+}^{n / 2} d x\right| \leq c \lambda^{n / 2}\left\{f^{-1}(\lambda)\right\}^{n-v_{n}}
$$

for large $\lambda$ where $v_{n}=1 /(2 n)(n \geq 3), v_{2}=v_{1}=1 / 8$.

By the assumption (4.1), we have

$$
N(\lambda) \sim(2 \pi)^{-n} \omega_{n} \int_{\boldsymbol{R}^{n}}(\lambda-V)_{+}^{n / 2} d x \quad \text { as } \lambda \rightarrow \infty .
$$

Q.E.D.

Remark 4.1. By Theorem 4.1, we can give the asymptotic formula for the Schrödinger operator $-\Delta+V$ on $\boldsymbol{R}^{n}$ when, for example,

$$
\begin{aligned}
& V(x)=\exp \exp \cdots \exp |x|, \\
& V(x)=|x|^{\alpha}(\alpha>0)
\end{aligned}
$$


or

$$
V(x)=\log \cdots \log |x|
$$

for large $|x|$. By easy calculations we can show that these potentials satisfy the condition (4.1).

Next we consider potentials which are not necessarily radial. First we shall give a theorem which is an extension of the result by Titchmarsh [17, p176].

Theorem 4.2. Let $V \in C^{1}\left(\mathbb{R}^{n}\right), V \geq 1$ and $V(x) \rightarrow \infty$ as $|x| \rightarrow \infty$. We assume the following conditions.

(4.2) $\lambda^{n / 2} \sigma(\lambda)=O(\Psi(\lambda))$ as $\lambda \rightarrow \infty$ where $\Psi(\lambda)=\int_{R^{n}}(\lambda-V)_{+}^{n / 2} d x$

(4.3) There exist constants $c_{1}>0$ and $a$ such that $0 \leq a<1 / 2$ and $|\nabla V(x)| \leq c_{1} V(x)^{1+a}$ for large $|x|$.

(4.4) $\lim _{\lambda \rightarrow \infty} \frac{A(\lambda)}{\sigma(\lambda)}=0$ where

$$
A(\lambda)=\left|\left\{x \in \mathbb{R}^{n}: \operatorname{dist}\left(x, \partial \Omega_{\lambda}\right) \leq \lambda^{-a}\right\}\right|,
$$

$\Omega_{\lambda}=\left\{x \in \mathbb{R}^{n}: V(x)<\lambda\right\}$ and $a$ is a constant in (4.3).

Then

$$
N(\lambda) \sim(2 \pi)^{-n} \omega_{n} \int_{R^{n}}(\lambda-V)_{+}^{n / 2} d x
$$

Proof. Let $\theta$ be a positive constant such that $a<\theta<1 / 2$. In Theorem 2.2 we set $l(\lambda)=\lambda^{-\theta}$ for large $\lambda$.

First we consider the condition (H1). By (4.4), we have

$$
\frac{\left(\#\left(J_{\lambda} \backslash I_{\lambda}\right)\right)}{\left(\# I_{\lambda}\right)} \leq \frac{A(\lambda)}{\sigma(\lambda)-A(\lambda)}=\frac{A(\lambda) \sigma(\lambda)^{-1}}{1-A(\lambda) \sigma(\lambda)^{-1}} \rightarrow 0
$$

as $\lambda \rightarrow \infty$.

Next we consider the condition $(\mathrm{H} 2)^{\prime}$. We have, for large $\lambda$, by (4.3), 


$$
\begin{aligned}
& \tilde{G}_{n}(V, \lambda)=l(\lambda)^{n\left(1-q_{n} / p_{n}\right)} \sum_{\zeta \in I_{\lambda}}\left(\int_{Q_{\zeta}}\left|V(x)-V_{Q_{\zeta}}\right|^{p_{n}} d x\right)^{q_{n} / p_{n}} \\
& \leq c l(\lambda)^{n\left(1-q_{n} / p_{n}\right)} l(\lambda)^{q_{n}} \sum_{\zeta \in I_{\lambda}}\left(\int_{Q_{\zeta}}|\nabla V(x)|^{p_{n}} d x\right)^{q_{n} / p_{n}} \\
& \leq c l(\lambda)^{n\left(1-q_{n} / p_{n}\right)} l(\lambda)^{q_{n}} c_{1}^{q_{n}} \lambda^{(1+a) q_{n}} \sum_{\zeta \in I_{\lambda}}\left|Q_{\zeta}\right|^{q_{n} / p_{n}} \\
& \leq c c_{1}^{q_{n}} \lambda^{q_{n}} \sigma(\lambda) \lambda^{-(\theta-a) q_{n}} .
\end{aligned}
$$

Therefore, by (4.2) and Theorem 2.2, we have

$$
N(\lambda) \sim(2 \pi)^{-n} \omega_{n} \int_{R^{n}}(\lambda-V)_{+}^{n / 2} d x
$$

Q.E.D.

When $n \geq 3$, we can prove the following new result.

Theorem 4.3. Let $n \geq 3$. Let $V \in C^{1}\left(\boldsymbol{R}^{n}\right), \quad V \geq 1$ and $V(x) \rightarrow \infty$ as $|x| \rightarrow \infty$. We assume the following conditions.

(4.5) There exists a positive constant $c_{1}$ such that $\sigma(2 \lambda) \leq c_{1} \sigma(\lambda)$ for large $\lambda$.

(4.6) There exist constants $c_{2}>0$ and $a$ such that $0 \leq a<1 / 2$ and $|\nabla V(x)| \leq c_{2} V(x)^{1+a}$ for large $|x|$.

Then

$$
N(\lambda) \sim(2 \pi)^{-n} \omega_{n} \int_{R^{n}}(\lambda-V)_{+}^{n / 2} d x
$$

Proof. Let $\theta$ be a positive constant such that $a<\theta<1 / 2$. In Theorem 2.3 we set $l(\lambda)=\lambda^{-\theta}$ for large $\lambda$. Clearly the conditions (C1) and (C2) are satisfied. We consider the conditions (C3) and (C4) in Theorem 2.3. We have

$$
\tilde{G}_{n}(V, \lambda)=\sum_{\zeta \in I_{\lambda}} \int_{Q_{\zeta}}\left|V-V_{Q_{\zeta}}\right|^{n / 2} d x \leq c \sum_{\zeta \in I_{\lambda}} l(\lambda)^{n / 2} \int_{Q_{\zeta}}|\nabla V(x)|^{n / 2} d x
$$

By the assumption (4.6), the last term does not exceed 


$$
c c_{2}^{n / 2} l(\lambda)^{n / 2} \lambda^{(1+a) n / 2} \sigma(\lambda)=c c_{2}^{n / 2} \lambda^{n / 2} \sigma(\lambda) \lambda^{-(\theta-a) n / 2} .
$$

Hence we have

$$
\tilde{G}_{n}(V, \lambda) \leq c c_{2}^{n / 2} \lambda^{n / 2} \sigma(\lambda) \lambda^{-(\theta-a) n / 2}
$$

for large $\lambda$ and the condition (C3) holds.

Next we consider the condition (C4). Let $\zeta \in J_{\lambda} \backslash I_{\lambda}$. For every $x \in Q_{\zeta} \cap \Omega_{\lambda}$ such that $V(x)<\lambda$, there is a $z \in \overline{Q_{\zeta}} \cap \overline{\Omega_{\lambda}}$ such that $V(z)=\lambda$ and the line segment which connect $x$ and $z$ lies in $\overline{Q_{\zeta}} \cap \overline{\Omega_{\lambda}}$. If there are no such $z$ and line segment, then we conclude that $V(y)<\lambda$ for all $y \in \overline{Q_{\zeta}}$, that is, $\zeta \in I_{\lambda}$ and this contradicts the choice of $\zeta$.

By (4.6), we have, for all $x \in Q_{\zeta} \cap \Omega_{\lambda}$ such that $V(x)<\lambda$,

$$
\begin{gathered}
\lambda-V(x)=V(z)-V(x) \leq \int_{0}^{1}|\nabla V(x+t(z-x)) \cdot(z-x)| d t \\
\leq c c_{2} l(\lambda) \lambda^{1+a} \leq c c_{2} \lambda^{1+a-\theta} .
\end{gathered}
$$

Hence the condition (C4) holds. Therefore, by Theorem 2.3, we have

$$
N(\lambda) \sim(2 \pi)^{-n} \omega_{n} \int_{\boldsymbol{R}^{n}}(\lambda-V)_{+}^{n / 2} d x \quad \text { as } \lambda \rightarrow \infty .
$$

Q.E.D.

Remark 4.2. The condition (4.2) is weaker than (4.5). In fact we can easily give an example of $V$ such that (4.2) holds and (4.5) is false.

Remark 4.3. In [4] Feigin studies the asymptotic distribution of eigenvalues of pseudo-differential operators which contain the Schrödinger operator $-\Delta+V$ where $V \in C^{\infty}\left(\boldsymbol{R}^{n}\right), V \geq 1$ and $\left|\partial^{\beta} V(x)\right| \leq C_{\beta} V(x)^{1+a|\beta|}$ for all $\beta \in N^{n}, x \in R^{n}$. In Theorem 4.3 our assumption on $V$ is $V \in C^{1}\left(R^{n}\right)$ and this is an improvement on the regularity condition in the Feigin's result.

Next we will give an alternative proof to Theorem 2 in Rozenbljum [12].

Theorem 4.4. Let $V \in L_{\mathrm{loc}}^{\infty}\left(\boldsymbol{R}^{n}\right), V \geq 1$ and $V(x) \rightarrow \infty$ as $|x| \rightarrow \infty$. We assume the following conditions. 
(4.7) There exists a positive constant $c_{1}$ such that $\sigma(2 \lambda) \leq c_{1} \sigma(\lambda)$ for large $\lambda$.

(4.8) Let $\Xi$ be a family of disjoint open unit cubes $\left\{Q_{i}\right\}$ such that $\boldsymbol{R}^{n}=\cup \overline{Q_{i}}$. There exist a continuous and decreasing function $v(t), t \in[1, \infty)$, $v(t) \rightarrow 0$ as $t \rightarrow \infty$, and an index $a, 0 \leq a \leq 1 / 2$ such that

$$
|V(x)-V(y)| \leq|x-y|^{2 a} V(x)^{1+a} v(V(x))
$$

for an arbitrary $Q_{i} \in \Xi$ and for all $x, y \in Q_{i}$.

Then

$$
N(\lambda) \sim(2 \pi)^{-n} \omega_{n} \int_{R^{n}}(\lambda-V)_{+}^{n / 2} d x
$$

Proof. First we consider the case $a>0$. Let $k(t)=t v(t)^{-1 /(2 a)}$ for $t \geq 1$. Then $k(t)$ is a strictly increasing function on $[1, \infty)$ and $k(t) \rightarrow \infty$ as $t \rightarrow \infty$. Let $h(s)$ be the inverse function of $k(t)$, that is, $h(s)=k^{-1}(s)$. In Theorem 2.3 we set $l(\lambda)=\left[h(\lambda)^{1 / 2}\right]^{-1}$ for sufficiently large $\lambda$ where $[x]$ denotes the integral part of $x$, and we consider cubes $\left\{Q_{\zeta}\right\}_{\zeta \in Z_{\lambda}}$ with side length $l(\lambda)$ which are partition of each unit cube in $\Xi$.

Then we have

$$
\lambda^{-1 / 2} l(\lambda)^{-1}=\lambda^{-1 / 2}\left[h(\lambda)^{1 / 2}\right] \leq v(h(\lambda))^{1 /(4 a)} \rightarrow 0
$$

as $\lambda \rightarrow \infty$. (4.9) means that the condition (C2) in Theorem 2.3 holds.

We will consider the condition (C3) in Theorem 2.3 when $n \geq 3$. We decompose $I_{\lambda}$ to disjoint two subsets $I_{\lambda}^{1}$ and $I_{\lambda}^{2}$, that is,

$$
I_{\lambda}^{1}=\left\{\zeta \in I_{\lambda} ; \inf _{x \in Q_{\zeta}} V(x) \geq h(\lambda)\right\} \text { and } I_{\lambda}^{2}=I_{\lambda} \backslash I_{\lambda}^{1}
$$

We shall estimate $\sum_{\zeta \in I_{\lambda}^{2}} \int_{Q_{\zeta}}\left|V-V_{Q_{\zeta}}\right|^{n / 2} d x . \quad$ For $\zeta \in I_{\lambda}^{2}$, there exists a $z \in Q_{\zeta}$ such that $V(z)<h(\lambda)$. Hence we have, for all $x \in Q_{\zeta}$ and large $\lambda$,

$$
|V(z)-V(x)| \leq \operatorname{cl}(\lambda)^{2 a} V(z)^{1+a} v(V(z)) \leq \operatorname{ch}(\lambda)^{-a} h(\lambda)^{1+a} v(1)=\operatorname{ch}(\lambda) .
$$

Accordingly we have $V(x) \leq \operatorname{ch}(\lambda) \leq c \lambda v(h(\lambda))^{1 /(2 a)}$ for all $x \in Q_{\zeta}, \zeta \in I_{\lambda}^{2}$ and

$$
\sum_{\zeta \in I_{\lambda}^{2}} \int_{Q_{\zeta}}\left|V-V_{Q_{\zeta}}\right|^{n / 2} d x \leq c \lambda^{n / 2} \sigma(\lambda) v(h(\lambda))^{n /(4 a)}
$$


we have

Next we shall estimate $\sum_{\zeta \in I_{\lambda}^{1}} \int_{Q_{\zeta}}\left|V-V_{Q_{\zeta}}\right|^{n / 2} d x$. By the condition (4.8),

$$
\begin{aligned}
|V(x)-V(y)| & \leq c l(\lambda)^{2 a} V(x)^{1+a} v(V(x)) \\
& \leq c \lambda^{-a} v(h(\lambda))^{-1 / 2} \lambda^{1+a} v(h(\lambda))=c \lambda v(h(\lambda))^{1 / 2}
\end{aligned}
$$

for $\zeta \in I_{\lambda}^{1}$ and for all $x, y \in Q_{\zeta}$.

Hence

$$
\sum_{\zeta \in I_{\lambda}^{1}} \int_{Q_{\zeta}}\left|V-V_{Q_{\zeta}}\right|^{n / 2} d x
$$

$$
\leq \sum_{\zeta \in I_{\lambda}^{1}} \int_{Q_{\zeta}}\left\{\frac{1}{\left|Q_{\zeta}\right|} \int_{Q_{\zeta}}|V(x)-V(y)| d y\right\}^{n / 2} d x \leq c \lambda^{n / 2} \sigma(\lambda) v(h(\lambda))^{n / 4} .
$$

By (4.10) and (4.11), we have

$$
\sum_{\zeta \in I_{\lambda}} \int_{Q_{\zeta}}\left|V-V_{Q_{\zeta}}\right|^{n / 2} d x \leq c \lambda^{n / 2} \sigma(\lambda) v(h(\lambda))^{n / 4}
$$

for large $\lambda$.

When $n=2$, similar arguments as in the case $n \geq 3$ give

$$
l(\lambda) \sum_{\zeta \in I_{\lambda}}\left(\int_{Q_{\zeta}}\left|V-V_{Q_{\zeta}}\right|^{2} d x\right)^{1 / 2} \leq c \lambda \sigma(\lambda) v(h(\lambda))^{1 / 2}
$$

When $n=1$, we have

$$
l(\lambda)^{3 / 4} \sum_{\zeta \in I_{\lambda}}\left(\int_{Q_{\zeta}}\left|V-V_{Q_{\zeta}}\right| d x\right)^{1 / 4} \leq c \lambda^{1 / 4} \sigma(\lambda) v(h(\lambda))^{1 / 8}
$$

for large $\lambda$. Hence the condition (C3) in Theorem 2.3 holds.

Finally we consider the condition (C4). Let $\zeta \in J_{\lambda} \backslash I_{\lambda}$. If $V$ is discontinuous at $\partial Q_{\zeta}$, then we define $\tilde{V}_{\zeta}$ as the function which is continuous on $\overline{Q_{\zeta}}$ and coincides with $V$ on $Q_{\zeta}$. $B y(4.8)$, we have

$$
\left|\tilde{V}_{\zeta}(x)-\tilde{V}_{\zeta}(y)\right| \leq|x-y|^{2 a} \tilde{V}_{\zeta}(x)^{1+a} v\left(\tilde{V}_{\zeta}(x)\right)
$$


for all $x, y \in \overline{Q_{\zeta}}$.

Since $\zeta \in J_{\lambda} \backslash I_{\lambda}$, there exists a $z \in \overline{Q_{\zeta}}$ such that $\tilde{V}_{\zeta}(z)=\lambda$. Hence we have, for all $x \in Q_{\zeta}$ and large enough $\lambda$,

$$
\begin{aligned}
& |\lambda-V(x)|=\left|\tilde{V}_{\zeta}(z)-\tilde{V}_{\zeta}(x)\right| \\
& \quad \leq \operatorname{cl}(\lambda)^{2 a} \tilde{V}_{\zeta}(z)^{1+a} v\left(\tilde{V}_{\zeta}(z)\right)=\operatorname{cl}(\lambda)^{2 a} \lambda^{1+a} v(\lambda) .
\end{aligned}
$$

By (4.9) we have $h(\lambda)<\lambda$ for large $\lambda$. Hence we have $v(\lambda) \leq v(h(\lambda))$ for sufficiently large $\lambda$.

Therefore we have, for all $x \in Q_{\zeta}$ and large $\lambda$,

$$
|\lambda-V(x)| \leq c \lambda^{-a} v(h(\lambda))^{-1 / 2} \lambda^{1+a} v(h(\lambda))=c \lambda v(h(\lambda))^{1 / 2}
$$

and the condition (C4) holds.

By Theorem 2.3 we have

$$
N(\lambda) \sim(2 \pi)^{-n} \omega_{n} \int_{\boldsymbol{R}^{n}}(\lambda-V)_{+}^{n / 2} d x \quad \text { as } \quad \lambda \rightarrow \infty .
$$

When $a=0$, we set $l(\lambda)=1$ and $h(\lambda)=\lambda^{1 / 2}$. Similar arguments as before gives the conclusion.

Q.E.D.

Next we consider the condition which is a modification of the result by Fleckinger [5] (cf. Fleckinger and Lapidus [6, Section 5]).

Theorem 4.5. Let $V$ be a continuous function on $\boldsymbol{R}^{n}, V \geq 1$ and $V(x) \rightarrow \infty$ as $|x| \rightarrow \infty$. We assume the following conditions.

(4.13) There exists a positive constant $c_{1}$ such that $\sigma(2 \lambda) \leq c_{1} \sigma(\lambda)$ for large $\lambda$.

(4.14) For every $\varepsilon>0$ there exists a $\eta>0$ such that $|V(x)-V(y)| \leq \varepsilon V(x)$ for all $x, y \in \boldsymbol{R}^{n},|x-y|<\eta$.

Then

$$
N(\lambda) \sim(2 \pi)^{-n} \omega_{n} \int_{\boldsymbol{R}^{n}}(\lambda-V)_{+}^{n / 2} d x
$$

Proof. In Theorem 2.3 we set $l(\lambda)=\lambda^{-\theta}$ where $0<\theta<1 / 2$. For every $\varepsilon>0$ we define $\eta(\varepsilon)$ as the maximal $\eta$ satisfying the condition (4.14). For $\lambda>0$ we set 


$$
\varepsilon(\lambda)=\inf \left\{\varepsilon>0 ; \eta(\varepsilon)>\sqrt{n} \lambda^{-\theta}\right\}
$$

It is clear that $\varepsilon(\lambda) \rightarrow 0$ as $\lambda \rightarrow \infty$. We have

$$
\tilde{G}_{n}(V, \lambda) \leq \lambda^{q_{n}} \sigma(\lambda) \varepsilon(\lambda)^{q_{n}}
$$

for sufficiently large $\lambda$ where $q_{n}$ is the constant in Theorem 2.2.

As in the proof of Theorem 4.4, we have, for all $x \in Q_{\zeta}\left(\zeta \in J_{\lambda} \backslash I_{\lambda}\right)$,

$$
|\lambda-V(x)| \leq \varepsilon(\lambda) \lambda
$$

Hence we have

$$
N(\lambda) \sim(2 \pi)^{-n} \omega_{n} \int_{\mathbb{R}^{n}}(\lambda-V)_{+}^{n / 2} d x .
$$

Q.E.D.

Remark 4.4. In [5] Fleckinger considers additional condition as follows:

(4.15) Consider a tessellation of $\mathbb{R}^{n}$ by a family of disjoint cubes $\left\{Q_{\zeta}\right\}_{\zeta \in Z}$ where $Z$ denotes an index set and $Q_{\zeta}$ is an open cube with side length $\eta>0$. We set

$$
I=\left\{\zeta \in Z: \overline{Q_{\zeta}} \subset \Omega_{\lambda}\right\}
$$

and

$$
J=\left\{\zeta \in Z: \overline{Q_{\zeta}} \cap \Omega_{\lambda} \neq \varnothing\right\}
$$

where $\Omega_{\lambda}=\left\{x \in \mathbb{R}^{n}: V(x)<\lambda\right\}$. Then

$$
\lim _{\eta \rightarrow 0} \frac{(\#(J \backslash I))}{(\# J)}=0
$$

for large $\lambda$.

In our proof we do not need this condition (4.15).

\section{$\S 5 . \quad$ Nonclassical Potentials}

In this section we consider the eigenvalue asymptotics of the Schrödinger operator $-\Delta+V$ on $\mathbb{R}^{n}$ when $V$ is a nonclassical potential. A nonclassical potential is the potential $V$ such that $V \geq 0$ and the set $\left\{x \in \mathbb{R}^{n}: V(x)=0\right\}$ is 
an unbounded set. Several results on these nonclassical potentials are known ([8], [9, Section 10] [11], [13], [14], [15]) and those are only on the potentials whose zero sets are cones in $\boldsymbol{R}^{n}$. Our method is a modification of that of Tachizawa [15] and we can apply it to some nonclassical potentials whose zero sets are not cones.

The potentials which we consider in this section belong to the special function class, that is, $A_{\infty}$-weights and we use some results about $A_{\infty}$-weights in Tachizawa [15, Section 2].

Let $V$ be a nonnegative continuous function on $R^{n}$ and let $T^{\prime}$ be the self-adjoint operator associated with the sesquilinear form

$$
t^{\prime}(u, v)=\int_{\boldsymbol{R}^{n}} \nabla u \overline{\nabla v} d x+\int_{\boldsymbol{R}^{n}} V u \bar{v} d x
$$

as in Section 2. We write $T^{\prime}=-\Delta+V$, formally. If $T^{\prime}$ has only discrete spectrum, then we denote the number of eigenvalues less than $\lambda$ as $N^{\prime}(\lambda)$.

We will consider some examples.

Example 5.1. $\quad V(x, y)=|x y|^{\alpha},(x, y) \in \boldsymbol{R}^{2}, \alpha>0$.

This potential is considered by Simon [13]. By Lemma 2.3 in [15], $V(x, y)=|x y|^{\alpha}$ is an $A_{\infty}$-weight on $\boldsymbol{R}^{2}$ and, by Remark 2.3 in [15], it is easily proved that the Schrödinger operator $T^{\prime}=-\Delta+|x y|^{\alpha}$ has only discrete spectrum.

We shall prove

$$
N^{\prime}(\lambda) \sim \frac{1}{\pi} \lambda^{1+1 / \alpha} \log \lambda \quad \text { as } \lambda \rightarrow \infty
$$

by the method of Tachizawa [15].

We set

$$
V_{1}(x, y)=|x y|^{\alpha}+1
$$

and let $T$ be the self-adjoint operator associated with $-\Delta+V_{1}$. Since $N(\lambda)$ denotes the number of eigenvalues of $T$ less than $\lambda$, we have

$$
N(\lambda)=N^{\prime}(\lambda+1)
$$

We will estimate $N(\lambda)$ using the method in the proof of Theorem 2.1. We set, for large $\lambda$, 


$$
\begin{gathered}
\Omega_{\lambda}=\left\{(x, y) \in \mathbb{R}^{2}:|x y|^{\alpha}+1<\lambda,|x|<c_{1}(\lambda-1)^{(2+\alpha) /(2 \alpha),}\right. \\
\left.|y|<c_{1}(\lambda-1)^{(2+\alpha) /(2 \alpha)}\right\}, \\
l(\lambda)=(\lambda-1)^{-1 / 2}(\log (\lambda-1))^{1 / 2}, \\
\tilde{I}_{\lambda}=\left\{\zeta \in Z_{\lambda}: \overline{Q_{\zeta}} \subset \Omega_{\lambda}\right\}
\end{gathered}
$$

and

$$
\tilde{J}_{\lambda}=\left\{\zeta \in Z_{\lambda}: \overline{Q_{\zeta}} \cap \Omega_{\lambda} \neq \varnothing\right\}
$$

where $c_{1}$ is a positive constant depending only on $\alpha$ and we shall give precise value of $c_{1}$ later. In the proof of Theorem 2.1 we used the fact that $n_{1}\left(\lambda^{-1}\right.$; $V, 1, D)=0$ where $D=\mathbb{R}^{n} \backslash \bigcup_{\zeta \in J} \overline{Q_{\zeta}}$. Hence the proof of Theorem 2.1 is valid in this nonclassical case if we prove $n_{1}\left(\lambda^{-1} ; V, 1, \Omega_{\lambda}^{*}\right)=0$ where

$$
\Omega_{\lambda}^{*}=\mathbb{R}^{2} \backslash\left(\bigcup_{\zeta \in \widetilde{J}} \overline{Q_{\zeta}}\right)
$$

We have

$$
\Omega_{\lambda}^{*}=\text { the interior of } \overline{F_{1} \cup F_{2} \cup F_{3}}
$$

where

$$
\begin{aligned}
& F_{1}=\left\{(x, y) \in \Omega_{\lambda}^{*}:|y|<c_{2}(\lambda-1)^{-1 / 2}\right\}, \\
& F_{2}=\left\{(x, y) \in \Omega_{\lambda}^{*}:|x|<c_{2}(\lambda-1)^{-1 / 2}\right\},
\end{aligned}
$$

and

$$
F_{3}=\Omega_{\lambda}^{*} \backslash \overline{\left(F_{1} \cup F_{2}\right)}
$$

$c_{2}$ is a positive constant which will be given later. We will show that $n_{1}\left(\lambda^{-1}\right.$; $\left.V, 1, \Omega_{\lambda}^{*}\right)=0$. If $\lambda$ is sufficiently large, then we can write

$$
F_{1}=\left\{(x, y) \in \mathbb{R}^{2}:|x|>k(\lambda),|y|<c_{2}(\lambda-1)^{-1 / 2}\right\}
$$

and

$$
F_{2}=\left\{(x, y) \in \mathbb{R}^{2}:|y|>k(\lambda),|x|<c_{2}(\lambda-1)^{-1 / 2}\right\}
$$

where $k(\lambda)$ is a function of $\lambda$ such that $k(\lambda) \geq c_{1}(\lambda-1)^{(2+\alpha) /(2 \alpha)}$.

Then, by the Dirichlet-Neumann bracketing method, 


$$
n_{1}\left(\lambda^{-1} ; V_{1}, 1, \Omega_{\lambda}^{*}\right) \leq \sum_{i=1}^{3} n_{1}\left(\lambda^{-1} ; V_{1}, 1, F_{i}\right)
$$

For the proof of $n_{1}\left(\lambda^{-1} ; V_{1}, 1, F_{i}\right)=0$ for $i=1,2$, we use the following lemma which is derived from Lemma 2.4 in [15].

Lemma 5.1. Let $\alpha>0$ and $A>0$. Then there exist positive constants $\kappa_{1}$ and $\kappa_{2}$ depending only on $\alpha$ such that

$$
\int_{I}\left\{\left|\frac{d u}{d x}\right|^{2}+A|x|^{\alpha}|u|^{2}\right\} d x \geq \kappa_{1} A^{2 /(2+\alpha)} \int_{I}|u|^{2} d x
$$

for all $u \in S_{1}(I)$ where $I=\left(-\kappa_{2} A^{-1 /(2+\alpha)}, \kappa_{2} A^{-1 /(2+\alpha)}\right)$ and $S_{1}(I)$ denotes the space defined in Section 2.

If we set $c_{1}=\max \left\{\kappa_{1}^{-(2+\alpha) /(2 \alpha)}, \kappa_{2}^{-(2+\alpha) / 2}\right\}+1$ and $c_{2}=c_{1}^{-\alpha /(2+\alpha)} \kappa_{2}$, then we have

$$
\begin{gathered}
\iint_{F_{1}}\left\{|\nabla u|^{2}+\left(|x y|^{\alpha}+1\right)|u|^{2}\right\} d x d y \\
\geq \int_{|x|>k(\lambda)} d x \int_{|y|<c_{2}(\lambda-1)^{-1 / 2}}\left\{\left|\frac{\partial u}{\partial y}\right|^{2}+c_{1}^{\alpha}(\lambda-1)^{(2+\alpha) / 2}|y|^{\alpha}|u|^{2}\right\} d y \\
+\iint_{F_{1}}|u|^{2} d x d y \\
>(\lambda-1) \iint_{F_{1}}|u|^{2} d x d y+\iint_{F_{1}}|u|^{2} d x d y=\lambda \iint_{F_{1}}|u|^{2} d x d y
\end{gathered}
$$

for all $u \in S_{1}\left(F_{1}\right), u \neq 0$ and for sufficiently large $\lambda$. Hence we have

$$
n_{1}\left(\lambda^{-1} ; V_{1}, 1, F_{1}\right)=0
$$

Similarly

$$
n_{1}\left(\lambda^{-1} ; V_{1}, 1, F_{2}\right)=0
$$

By the choice of $c_{2}$, we can show

$$
V_{1}(x, y)>\lambda \text { for all }(x, y) \in F_{3} .
$$


As a result

$$
n_{1}\left(\lambda^{-1} ; V_{1}, 1, F_{3}\right)=0 \text {. }
$$

By (5.3), we have

$$
n_{1}\left(\lambda^{-1} ; V_{1}, 1, \Omega_{\lambda}^{*}\right)=0
$$

if $\lambda$ is sufficiently large.

Now we consider the conditions $(\mathrm{H} 1)$ and $(\mathrm{H} 2)$. Simple calculations give, for large $\lambda$,

$$
\frac{\left(\#\left(\tilde{J}_{\lambda} \backslash \tilde{I}_{\lambda}\right)\right)}{\left(\# \tilde{I}_{\lambda}\right)} \leq c_{3}(\log (\lambda-1))^{-1 / 2}
$$

and

$$
l(\lambda) \sum_{\zeta \in \tilde{I}_{\lambda}}\left\|g-g_{\zeta}\right\|_{2, Q_{\zeta}} \leq c_{4}\left|\Omega_{\lambda}\right|(\log (\lambda-1))^{-1 / 4}
$$

where $g=1-\left(|x y|^{\alpha}+1\right) \lambda^{-1}, g_{\zeta}=\left|Q_{\zeta}\right|^{-1} \int_{Q_{\zeta}} g d x$ and $c_{3}, c_{4}$ are positive constants not depending on $\lambda$. Applying the proof of Theorem 2.1, we have, for large $\lambda$,

$$
\left|N(\lambda)-\frac{1}{4 \pi} \int_{\Omega_{\lambda}}\left(\lambda-|x y|^{\alpha}-1\right) d x d y\right| \leq c_{5} \lambda\left|\Omega_{\lambda}\right|(\log (\lambda-1))^{-1 / 8}
$$

where $c_{5}$ is a positive constant not depending on $\lambda$. Hence, by (5.2), we have

$$
\left|N^{\prime}(\lambda)-\frac{1}{4 \pi} \int_{\Omega_{\lambda}^{\prime}}\left(\lambda-|x y|^{\alpha}\right) d x d y\right| \leq c_{7}(\lambda+1)\left|\Omega_{\lambda}^{\prime}\right|(\log \lambda)^{-1 / 8}
$$

where

$$
\Omega_{\lambda}^{\prime}=\left\{(x, y) \in \boldsymbol{R}^{2}:|x y|^{\alpha}<\lambda,|x|<c_{1} \lambda^{(2+\alpha) /(2 \alpha)},|y|<c_{1} \lambda^{(2+\alpha) /(2 \alpha)}\right\}
$$

We can easily show that the order of $\int_{\Omega_{\lambda}^{\prime}}\left(\lambda-|x y|^{\alpha}\right) d x d y$ is same as that of $\lambda\left|\Omega_{\lambda}^{\prime}\right|$ as $\lambda \rightarrow \infty$. Hence we have

$$
N^{\prime}(\lambda) \sim \frac{1}{4 \pi} \int_{\Omega_{\lambda}^{\prime}}\left(\lambda-|x y|^{\alpha}\right) d x d y
$$


and simple calculation gives

Hence we have (5.1).

$$
\frac{1}{4 \pi} \int_{\Omega_{\lambda}^{\prime}}\left(\lambda-|x y|^{\alpha}\right) d x d y \sim \frac{1}{\pi} \lambda^{1+1 / \alpha} \log \lambda .
$$

Example 5.2. $V(x, y)=(1+|x|)^{\alpha}(1+|y|)^{\beta}|x|^{\gamma}|y|^{\delta}, x \in \boldsymbol{R}^{n_{1}}, y \in \boldsymbol{R}^{n_{2}}, \alpha, \beta, \gamma$, $\delta \geq 0, \alpha+\gamma \neq 0, \beta+\delta \neq 0, \gamma n_{2} \leq(\beta+\delta) n_{1}$ and $\delta n_{1} \leq(\alpha+\gamma) n_{2}$.

This potential is considered by Tachizawa [15]. In exactly the same way as in Example 5.1, we have

$$
N^{\prime}(\lambda) \sim(2 \pi)^{-n} \omega_{n} \int_{\Omega_{\lambda}^{\prime}}(\lambda-V)^{n / 2} d x d y \text { as } \lambda \rightarrow \infty
$$

where

$$
\begin{array}{r}
n=n_{1}+n_{2}, \\
\Omega_{\lambda}^{\prime}=\left\{(x, y) \in R^{n_{1}} \times R^{n_{2}}: V(x, y)<\lambda,|x|<c_{1} \lambda^{(2+\delta) /(2(\alpha+\gamma))},\right. \\
\left.|y|<c_{2} \lambda^{(2+\gamma) /(2(\beta+\delta))}\right\}
\end{array}
$$

and $c_{1}, c_{2}$ depend only on $\alpha, \beta, \gamma, \delta, n_{1}$ and $n_{2}$. Hence, in the case $\gamma n_{2}<(\beta+\delta) n_{1}$ and $\delta n_{1}<(\alpha+\gamma) n_{2}$, the order of $N^{\prime}(\lambda)$ is same as that of $\lambda^{v_{1}}+\lambda^{\dot{v_{2}}}$ and in the other cases, the order of $N^{\prime}(\lambda)$ is same as that of $\left(\lambda^{v_{1}}+\lambda^{v_{2}}\right) \log \lambda$ where $v_{1}=n / 2+n_{1} /(\alpha+\gamma)$ and $v_{2}=n / 2+n_{2} /(\beta+\delta)$.

For the proof of (5.4), we also set $l(\lambda)=(\lambda-1)^{-1 / 2}(\log (\lambda-1))^{1 / 2}$.

Example 5.3. $\quad V(x, y)=\Pi_{i=1}^{p}\left|x-a_{i}\right|^{\alpha_{i}} \cdot \Pi_{j=1}^{q}\left|y-b_{j}\right|^{\beta_{j}}, \quad(x, \quad y) \in \boldsymbol{R}^{2}$, $a_{1}<\cdots<a_{p}, \quad b_{1}<\cdots<b_{q}, \quad 0<\alpha_{i}, \quad 0<\beta_{j}, \quad \alpha_{i} \leq \sum_{j=1}^{q} \beta_{j}(i=1, \cdots, p), \quad \beta_{j} \leq \sum_{i=1}^{p} \alpha_{i}$ $(j=1, \cdots q)$.

Let $s_{1}=\max _{1 \leq i \leq p} \alpha_{i}, s_{2}=\max _{1 \leq j \leq q} \beta_{j}, \sigma_{1}=\sum_{i=1}^{p} \alpha_{i}$ and $\sigma_{2}=\sum_{j=1}^{q} \beta_{j}$. Let

$$
\mu_{1}=\left(2+s_{2}\right)\left(2 \sigma_{1}\right)^{-1}, \mu_{2}=\left(2+s_{1}\right)\left(2 \sigma_{2}\right)^{-1}
$$

and

$$
\Omega_{\lambda}^{\prime}=\left\{(x, y) \in \boldsymbol{R}^{2}: V(x, y)<\lambda,|x|<c_{1} \lambda^{\mu_{1}},|y|<c_{2} \lambda^{\mu_{2}}\right\}
$$


Then we have

$$
N^{\prime}(\lambda) \sim \frac{1}{4 \pi} \int_{\Omega_{\lambda}^{\prime}}(\lambda-V) d x d y \quad \text { as } \quad \lambda \rightarrow \infty
$$

and accordingly the order of $N^{\prime}(\lambda)$ as $\lambda \rightarrow \infty$ is same as that of

$$
\begin{array}{r}
\lambda^{1+1 / \sigma_{1}}\left\{\int_{\lambda^{-1 / 2}}^{1} t^{-s_{2} / \sigma_{1}} d t+\int_{1}^{\lambda^{1 / \sigma_{2}}} t^{-\sigma_{2} / \sigma_{1}} d t\right\} \\
+\lambda^{1+1 / \sigma_{2}}\left\{\int_{\lambda^{-1 / 2}}^{1} t^{-s_{1} / \sigma_{2}} d t+\int_{1}^{\lambda^{1 / \sigma_{1}}} t^{-\sigma_{1} / \sigma_{2}} d t\right\} .
\end{array}
$$

The proof of (5.5) is similar to that of (5.1). For the proof, we also set $l(\lambda)=(\lambda-1)^{-1 / 2}(\log (\lambda-1))^{1 / 2}$.

\section{References}

[1] Birman, M.S. and Solomyak, M.Z., Quantitative analysis in Sobolev imbedding theorems and applications to spectral theory, Amer. Math. Soc. Transl., 114 (1980), 1-132.

[2] Edmunds, D.E. and Evans, W.D., On the distribution of eigenvalues of Schrödinger operators, Arch. Rational Mech. Anal., 89 (1985), 135-167.

[3] - Spectral theory and differential operators, Clarendon Press, Oxford, 1987.

[4] Feigin, V.I., Asymptotic distribution of eigenvalues for hypoelliptic systems in $\mathbb{R}^{n}$, Math. USSR-Sb., 28 (1976), 533-552.

[5] Fleckinger, J., Estimate of the number of eigenvalues for an operator of Schrödinger type, Proc. Roy. Soc. Edinburgh Sect. A, 89 (1981), 355-361.

[6] Fleckinger, J. and Lapidus, M.L., Eigenvalues of elliptic boundary value problems with an indefinite weight functions, Trans. Amer. Math. Soc., 295 (1986), 305-324.

[ 7 ] — Remainder estimates for the asymptotics of elliptic eigenvalue problems with indefinite weights, Arch. Rational Mech. Anal., 98 (1987), 329-356.

[ 8 ] Gurarie, D., Nonclassical eigenvalue asymptotics for operators of Schrödinger type, Bull. Amer. Math. Soc., 15 (1986), 233-237.

[9] Levendorskii, S.Z., The approximate spectral projection method, Acta Appl. Math., 7 (1986), 137-197.

[10] Reed, M. and Simon, B., Methods of modern mathematical physics, Vol. IV: Analysis of operators, Academic Press, New York, 1978.

[11] Robert, D., Comportment asymptotique des valeurs propres d'operateurs du type Schrödinger a potential "dégénéré", J. Math. Pures Appl., 61 (1982), 275-300.

[12] Rozenbljum, G.V., Asymptotics of the eigenvalues of the Schrödinger operator, Math. USSR-Sb., 22 (1974), 349-371.

[13] Simon, B., Nonclassical eigenvalue asymptotics, J. Funct. Anal., 53 (1983), 84-98.

[14] Solomyak, M.Z., Asymptotics of the spectrum of the Schrödinger operator with nonregular homogeneous potential, Math. USSR-Sb., 55 (1986), 19-37.

[15] Tachizawa, K., Asymptotic distribution of eigenvalues of Schrödinger operators with nonclassical potentials, Tôhoku Math. J., 42 (1990), 381-406. 
[16] Tamura, H., Asymptotic formulas with sharp remainder estimates for eigenvalues of elliptic operators of second order, Duke Math. J., 49 (1982), 87-119.

[17] Titchmarsh, E.C., Eigenfunction expansions associated with second-order differential equations, Part II, Oxford University Press, Oxford, 1958.

[18] Wet, J.S.de and Mandl, F., On the asymptotic distribution of eigenvalues, Proc. Roy. Soc. Lond. A, 200 (1950), 572-580. 
